\title{
A Fredholm Determinant Identity and the Convergence of Moments for Random Young Tableaux
}

\author{
Jinho Baik, ${ }^{* \dagger}$ Percy Deift $\ddagger \S$ and Eric M. Rains $₫$
}

February 1, 2008

\begin{abstract}
We obtain an identity between Fredholm determinants of two kinds of operators, one acting on functions on the unit circle and the other acting on functions on a subset of the integers. This identity is a generalization of an identity between a Toeplitz determinant and a Fredholm determinant that has appeared in the random permutation context. Using this identity, we prove, in particular, convergence of moments for arbitrary rows of a random Young diagram under Plancherel measure.
\end{abstract}

\section{Introduction}

In [3], the authors considered the length $\ell_{N}(\pi)$ of the longest increasing subsequence of a random permutation $\pi \in S_{N}$, the symmetric group on $N$ numbers. They showed, in particular, that for $\tilde{\ell}_{N}(\pi):=\frac{\ell_{N}(\pi)-2 \sqrt{N}}{N^{1 / 6}}$

$$
\lim _{N \rightarrow \infty} \mathbb{P}\left(\tilde{\ell}_{N} \leq x\right)=F^{(1)}(x),
$$

where $F^{(1)}(x)$ is the Tracy-Widom distribution [36] for the largest eigenvalue of a random matrix from the Gaussian Unitary Ensemble (GUE). The authors also proved the convergence of moments,

$$
\lim _{N \rightarrow \infty} \mathbb{E}\left(\left(\tilde{\ell}_{N}\right)^{m}\right)=\int_{-\infty}^{\infty} x^{m} d F^{(1)}(x), \quad m=1,2, \cdots
$$

The authors then reinterpreted (1.1), (1.2) in terms of Young diagrams $\lambda=\left(\lambda_{1}, \lambda_{2}, \cdots\right)$ via the Robinson-Schensted correspondence. Here $\lambda_{j}$ is the number of boxes in the $j$ th row of $\lambda$ and $\lambda_{1} \geq$

*Department of Mathematics, Princeton University, Princeton, New Jersey, 08544, jbaik@math.princeton.edu

${ }^{\dagger}$ Institute for Advanced Study, Princeton, New Jersey 08540

$\ddagger$ University of Pennsylvania, Philadelphia, 19104, deift@math.upenn.edu

$\S$ Courant Institute of Mathematical Sciences, New York 10012

『AT\&T Research, New Jersey, Florham Park, New Jersey 07932, rains@research.att.com 
$\lambda_{2} \geq \cdots \geq 0$. The set of Young diagrams $Y_{N}$ of size $N, \sum_{j} \lambda_{j}=N$, is equipped with Plancherel measure,

$$
\mathbb{P}_{N}^{\mathrm{Plan}}(\lambda):=\frac{d_{\lambda}^{2}}{N !}, \quad \lambda \in Y_{N}
$$

where $d_{\lambda}$ is the number of standard Young tableaux of shape $\lambda$. Set

$$
\xi_{j}:=\frac{\lambda_{j}-2 \sqrt{N}}{N^{1 / 6}}, \quad j=1,2, \cdots
$$

Then (1.1), (1.2) imply that $\xi_{1}$ converges in distribution, together with all its moments, to $F^{(1)}$. This reinterpretation led the authors to conjecture that for all $k, \xi_{1}, \xi_{2}, \cdots, \xi_{k}$ converge to the joint distribution function $F\left(x_{1}, x_{2}, \cdots, x_{k}\right)$ for the first $k$ eigenvalues of a random GUE matrix. In 画, the authors verified the convergence in distribution, together with its moments, to the Tracy-Widom distribution $F^{(2)}$ for the second largest eigenvalue of a random GUE matrix. The conjecture for $\xi_{1}, \xi_{2}, \cdots, \xi_{k}$ was then proved in three independent papers [29], [8], [24], all appearing within a few months in the spring of 1999. Let $y_{j}$ be the $j$ th largest eigenvalue of a random $N \times N$ matrix from GUE with probability density

$$
d \mathbb{P}_{N}^{\mathrm{GUE}}\left(y_{1}, \cdots, y_{N}\right)=\frac{1}{Z_{N}} \prod_{1 \leq i<j \leq N}\left(y_{i}-y_{j}\right)^{2} \prod_{j=1}^{N} e^{-y_{j}^{2}} d y_{1} \cdots d y_{N}
$$

where $y_{1} \geq \cdots \geq y_{N}$, and $Z_{N}$ is the normalization constant. At the 'edge' of the spectrum, the following convergence in distribution is well-known (see, e.g. [36], [24] Theorem 1.4): for any $k \in \mathbb{N}$, there is a distribution function $F\left(x_{1}, \cdots, x_{k}\right)$ on $x_{1} \geq \cdots \geq x_{k}$ such that

$$
\lim _{N \rightarrow \infty} \mathbb{P}_{N}^{\mathrm{GUE}}\left(\left(y_{1}-\sqrt{2 N}\right) \sqrt{2} N^{1 / 6} \leq x_{1}, \cdots,\left(y_{k}-\sqrt{2 N}\right) \sqrt{2} N^{1 / 6} \leq x_{k}\right)=F\left(x_{1}, \cdots, x_{k}\right) .
$$

In all three papers [29, [8], [24], the authors showed that for any $x_{1}, \cdots, x_{k} \in \mathbb{R}^{k}$,

$$
\lim _{N \rightarrow \infty} \mathbb{P}_{N}^{\text {Plan }}\left(\xi_{1} \leq x_{1}, \cdots, \xi_{k} \leq x_{k}\right)=F\left(x_{1}, \cdots, x_{k}\right),
$$

but the question of the convergence of moments was left open.

Introduce the Poissonized Plancherel measure

$$
\mathbb{P}_{t}^{\text {Pois }}(\lambda)=\sum_{N=0}^{\infty} \frac{e^{-t^{2}} t^{2 N}}{N !} \mathbb{P}_{N}^{\text {Plan }}(\lambda), \quad t>0,
$$

on all Young diagrams, which corresponds to choosing $N$ as a Poisson variable with parameter $t^{2}$. Here $\mathbb{P}_{N}^{\text {Plan }}(\lambda)=0$ if $\lambda$ is not a partition of $N$. Throughout the paper, we will work with $\mathbb{P}_{t}^{\text {Pois }}(\lambda)$ rather than $\mathbb{P}_{N}^{\text {Plan }}(\lambda)$ itself. This is because the expectation with respect to $\mathbb{P}_{t}^{\text {Pois }}(\lambda)$ leads to convenient determinantal formulae. Indeed, in [19], Gessel proved the following formula

$$
\mathbb{P}_{t}^{\text {Pois }}\left(\lambda_{1} \leq n\right)=e^{-t^{2}} \operatorname{det}\left(\mathrm{T}_{\mathrm{n}}\right),
$$


where $\mathrm{T}_{\mathrm{n}}$ is the $n \times n$ Toeplitz matrix with entries $\left(\mathrm{T}_{\mathrm{n}}\right)_{p q}=c_{p-q}, 0 \leq p, q<n$, where $c_{k}$ is the $k^{t h}$ Fourier coefficient of $e^{t\left(z+z^{-1}\right)}, c_{k}=\int_{|z|=1} z^{-k} e^{t\left(z+z^{-1}\right)} \frac{d z}{2 \pi i z}$. This formula played a basic role in [3] in

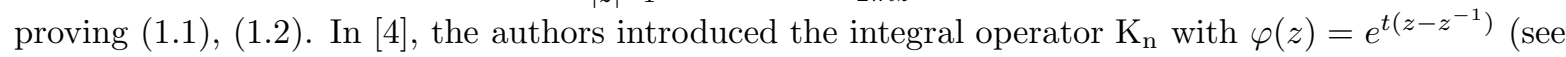
(2.1) below) and proved the following formulae

$$
\mathbb{P}_{t}^{\text {Pois }}\left(\lambda_{1} \leq n\right)=2^{-n} \operatorname{det}\left(1-\mathrm{K}_{\mathrm{n}}\right)
$$

and

$$
\mathbb{P}_{t}^{\text {Pois }}\left(\lambda_{2} \leq n+1\right)=\mathbb{P}_{t}^{\text {Pois }}\left(\lambda_{1} \leq n\right)+\left.\left(-\frac{\partial}{\partial s}\right)\right|_{s=1}\left[(1+\sqrt{s})^{-n} \operatorname{det}\left(1-\sqrt{s} \mathrm{~K}_{\mathrm{n}}\right)\right] .
$$

These formulae played a basic role in [4] in proving the analogue of (1.1), (1.2) for $\lambda_{2}$. In [8] and [24], and also later, in greater generality, in [28] and [31], the authors obtained the following identity : Let $\Lambda_{k}$ denote the (finite) set $\left\{n \in\{0,1, \cdots\}^{k}: \sum_{j=1}^{r} n_{j} \leq r-1, r=1, \cdots, k\right\}$. Then for $a_{k} \leq \cdots \leq a_{1} \leq$ $a_{0}=\infty$

$$
\begin{aligned}
\mathbb{P}_{t}^{\text {Pois }} & \left(\lambda_{1}-1 \leq a_{1}, \lambda_{2}-2 \leq a_{2}, \cdots, \lambda_{k}-k \leq a_{k}\right) \\
& =\left.\sum_{n \in \Lambda_{k}} \frac{1}{n_{1} ! \cdots n_{k} !} \frac{\partial^{|n|}}{\partial s_{1}^{n_{1}} \cdots \partial s_{k}^{n_{k}}}\right|_{s_{1}=\cdots=s_{k}=-1} \operatorname{det}\left(1+\left(\sum_{l=1}^{k} s_{l} \chi_{\left(a_{l}, a_{l-1}\right]}\right) S\right),
\end{aligned}
$$

where the matrix elements of $\mathrm{S}(i, j)$ are given in (2.3) below with $\varphi(z)=e^{\sqrt{\gamma\left(z-z^{-1}\right)}}$. As usual, $\chi_{(a, b]}$ denotes the characteristic function of the interval $(a, b]$, and so $\left(\sum_{l=1}^{k} s_{l} \chi_{\left(a_{l}, a_{l-1}\right]}\right) \mathrm{S}$ denotes the operator in $\ell^{2}(\mathbb{Z})$ with kernel $s_{l} \mathrm{~S}(i, j)$ if $i \in\left(a_{l}, a_{l-1}\right]$, and zero otherwise. Setting $a_{j}=2 t+x_{j} t^{1 / 3}, x_{1} \geq x_{2} \geq$ $\cdots \geq x_{k}$, and letting $t \rightarrow \infty$, and de-Poissonizing as in [26], the authors in [8] and [24] obtain (1.7). In [8] and [24], however, the authors are not able to prove convergence of moments. The reason for this is that it is possible to use the classical steepest-descent method to control $\operatorname{det}\left(1+\left(\sum_{l=1}^{k} s_{l} \chi_{\left(a_{l}, a_{l-1}\right]}\right) \mathrm{S}\right)$ for $a_{j}=2 t+x_{j} t^{1 / 3}$ as $t \rightarrow \infty$, uniformly for $x_{1} \geq x_{2} \geq \cdots \geq x_{k} \geq M$ for any fixed $M$. But as the $x_{j}$ 's tends to $-\infty$, the method break down. On the other hand, the authors in [3, 国 are able to control the lower tails of the probability distributions, and hence prove the convergence of moments for $\lambda_{1}$ and $\lambda_{2}$, using the steepest-descent method for the Riemann-Hilbert problem (RHP) naturally associated with $\mathrm{T}_{\mathrm{n}}$ and $\mathrm{K}_{\mathrm{n}}$ above. The steepest-descent method for RHP was introduced in [16], and extended to include fully non-linear oscillations in [15. The asymptotic analysis in [3], 传 is closely related to the analysis in 13, 14]. The main motivation for this paper was to find a formula for the joint distribution of $\lambda_{1}, \cdots, \lambda_{k}$, which generalized (1.11), and to which the above Riemann-Hilbert steepest-descent methods could be applied to obtain the lower tail estimates.

Note that from (1.9), (1.10) and (1.12), we have three formulae for the distribution of $\lambda_{1}$,

$$
\begin{aligned}
\mathbb{P}_{t}^{\text {Pois }}\left(\lambda_{1} \leq n\right) & =e^{-t^{2}} \operatorname{det}\left(\mathrm{T}_{\mathrm{n}}\right) \\
& =2^{-n} \operatorname{det}\left(1-\mathrm{K}_{\mathrm{n}}\right) \\
& =\operatorname{det}\left(1-\chi_{[n, \infty)} \mathrm{S}\right)
\end{aligned}
$$


and from (1.11) and (1.12), two formulae for the distribution of $\lambda_{2}$,

$$
\begin{aligned}
\mathbb{P}_{t}^{\text {Pois }}\left(\lambda_{2} \leq n+1\right) & =\mathbb{P}_{t}^{\text {Pois }}\left(\lambda_{1} \leq n\right)+\left.\left(-\frac{\partial}{\partial s}\right)\right|_{s=1}\left[(1+\sqrt{s})^{-n} \operatorname{det}\left(1-\sqrt{s} \mathrm{~K}_{\mathrm{n}}\right)\right] \\
& =\mathbb{P}_{t}^{\text {Pois }}\left(\lambda_{1} \leq n\right)+\left.\left(\frac{\partial}{\partial s}\right)\right|_{s=-1} \operatorname{det}\left(1+s \chi_{[n, \infty)} \mathrm{S}\right) .
\end{aligned}
$$

To obtain the second formula, we use the fact that $\Lambda_{k=2}=\{(0,0),(0,1)\}$ and set $a_{1}=\infty, a_{2}=n-1$ in (1.12). From (1.14), we might guess that

$$
(1+\sqrt{s})^{-n} \operatorname{det}\left(1-\sqrt{s} \mathrm{~K}_{\mathrm{n}}\right)=\operatorname{det}\left(1-s \chi_{[n, \infty)} \mathrm{S}\right) .
$$

The content of Theorem 2.1 is that precisely this relation is true for a general class of functions $\varphi(z)$, provided $\varphi(z)$ has no winding. If the winding number of $\varphi$ is non-zero, the above relation must be modified slightly as in (2.7). The fact that $e^{-t^{2}} \operatorname{det}\left(\mathrm{T}_{\mathrm{n}}\right)=\operatorname{det}\left(1-\chi_{[n, \infty)} \mathrm{S}\right)$ for (essentially) the same general class of $\varphi^{\prime} s$ (with zero winding number) was first proved in [7], with an alternative proof given in [5]. The relation (1.15) for general $s$ was proved essentially simultaneously with the present paper by Rains in [31], for a subclass of functions $\varphi$ with zero winding, using algebraic methods (see Remark 4 in Section 2). A particularly simple proof of the relation $e^{-t^{2}} \operatorname{det}\left(T_{n}\right)=\operatorname{det}\left(1-\chi_{[n, \infty)} S\right)$ can be found in the recent paper 9] of Böttcher (see also [10]). The paper [9] also extends Theorem 2.1 and 2.12 to the matrix case (see Remark 2.3 and 2.13 below).

In this paper, we will prove a general identity between determinants of operators of two types : the operators of the first type act on functions on the unit circle, and the operator of the second type act on functions on a subset of the integers. Specializations of this identity have, in particular, the following consequences :

(S1) A proof of the convergence of moments for $\xi_{1}, \cdots, \xi_{k}$ (see Theorem 3.1)

(S2) An interpretation of $F\left(x_{1}, \cdots, x_{k}\right)$ in (1.7) as a "multi-Painlevè" function (see Section 6). As we will see, the behavior of multi-Painlevé functions has similarities to the interactions of solitons in the classical theory of the Korteweg de Vries equation.

(S3) The analogue of Theorem 3.1 for signed permutations and so-called colored permutations (see Section (1)

(S4) New formulae for random word problems, certain 2-dimensional growth models, and also the so-called "digital boiling" model (see Section 7 )

The new identity is given in Theorem 2.1 in two closely related forms (2.7), 2.8). In (S1)-(S4), we only use (2.7).

As we will see, some simple estimates together with a Riemann-Hilbert analysis of $\operatorname{det}\left(1-\sqrt{s} \mathrm{~K}_{\mathrm{n}}\right)$ is enough to control the lower tail estimation of $\mathbb{P}_{t}^{\text {Pois }}(\lambda)$. The relation (1.15) generalizes to the multiinterval case, as described in Theorem 2.12 in Section 2. 
In Section 2, we prove the main identity (2.7), 2.8) in the single interval case, and also the identity (2.54) in the multi-interval case. In Section 3, we use (2.7) to prove the convergence of moments for random Young tableaux (Theorem 3.1). A stronger version of this result is given in (3.2). Section 14 contains certain tail estimates, needed in Section 3. Various estimates needed in Section 4 for a ratio of determinants are derived in Section 5 using the steepest-descent method for RHP's. In Section 6 , we introduce the notion of a multi-Painleve solution, and in Section 0 , we prove various formulae for colored permutations and also discuss certain random growth models from the perspective of Theorem 2.1 .

Acknowledgments. The authors would like to thank Xin Zhou for useful comments. The authors would also like to thank Albrecht Böttcher for pointing out a calculational error in an earlier version of the text. The work of the first author was supported in part by NSF Grant \# DMS 97-29992. The work of the second author was supported in part by NSF Grant \# DMS 00-03268, and also by the Guggenheim Foundation.

\section{Fredholm determinant identity}

Let $\varphi(z)$ be a continuous, complex-valued, non-zero function on the unit circle $\Sigma=\{z \in \mathbb{C}:|z|=1\}$. Define $K_{\mathrm{n}}$ to be the integral operator acting on $L^{2}(\Sigma, d w)$ with kernel

$$
\mathrm{K}_{\mathrm{n}}(z, w)=\frac{1-z^{n} \varphi(z) w^{-n} \varphi(w)^{-1}}{2 \pi i(z-w)}, \quad\left(\mathrm{K}_{\mathrm{n}} f\right)(z)=\int_{|w|=1} \mathrm{~K}_{\mathrm{n}}(z, w) f(w) d w .
$$

For a function $f$ on $\Sigma$, its Fourier coefficients are denoted by $f_{j}$, so that

$$
f(z)=\sum_{j \in \mathbb{Z}} f_{j} z^{j} .
$$

Let $\mathrm{S}$ be the matrix with entries

$$
\mathrm{S}(i, j)=\sum_{k \geq 1}\left(\varphi^{-1}\right)_{i+k} \varphi_{-j-k}, \quad i, j \in \mathbb{Z},
$$

and let $\mathrm{R}$ be the matrix with entries

$$
\mathrm{R}(i, j)=\sum_{k \leq 0}\left(\varphi^{-1}\right)_{i+k} \varphi_{-j-k}, \quad i, j \in \mathbb{Z},
$$

Let $\mathrm{S}_{\mathrm{n}}$ denote the operator $\chi_{[n, \infty)} \mathrm{S}$ acting on $\ell^{2}(\{n, n+1, \cdots\})$,

$$
\left(\mathrm{S}_{\mathrm{n}} f\right)(i)=\sum_{j \geq n} \mathrm{~S}_{\mathrm{n}}(i, j) f(j), \quad i \geq n,
$$

and let $\mathrm{R}_{\mathrm{n}}$ denote the operator $\chi_{(-\infty, n-1]} \mathrm{R}$ acting on $\ell^{2}(\{\cdots, n-2, n-1\})$,

$$
\left(\mathrm{R}_{\mathrm{n}} f\right)(i)=\sum_{j \leq n-1} \mathrm{R}_{\mathrm{n}}(i, j) f(j), \quad i \leq n-1 .
$$


Theorem 2.1. Let $\varphi(z)$ be a non-zero function on the unit circle satisfying $\sum_{j \in \mathbb{Z}}\left|j \varphi_{j}\right|<\infty$, which has winding number equal to $\#(\varphi)$. For $s \in \mathbb{C}$ and $n \in \mathbb{Z}, \mathrm{K}_{\mathrm{n}}, \mathrm{S}_{\mathrm{n}}$ and $\mathrm{R}_{\mathrm{n}}$ are trace class on $L^{2}(\Sigma, d w)$, $\ell^{2}(\{n, n+1, \cdots\})$ and $\ell^{2}(\{\cdots, n-1\})$ respectively, and we have

$$
\begin{aligned}
\operatorname{det}\left(1-s \mathrm{~K}_{\mathrm{n}}\right) & =(1+s)^{n+\#(\varphi)} \operatorname{det}\left(1-s^{2} \mathrm{~S}_{\mathrm{n}}\right), \quad s \neq-1, \\
& =(1-s)^{-n-\#(\varphi)} \operatorname{det}\left(1-s^{2} \mathrm{R}_{\mathrm{n}}\right), \quad s \neq 1 .
\end{aligned}
$$

Remark 2.2. Standard Banach algebra estimates show that if the winding number of $\varphi$ is zero and $\sum\left|j \varphi_{j}\right|<\infty$, then $\|\log \varphi\|_{\infty}+\left(\sum\left|j \|(\log \varphi)_{j}\right|^{2}\right)^{1 / 2}<\infty$. This is enough to prove that the first and the third terms in (1.13) are equal for all such $\varphi$ 's (see [5]). In particular, by (1.13), (2.7) is true for all $\varphi$ without winding and satisfying $\sum\left|j \varphi_{j}\right|<\infty$, when $s=1$.

Remark 2.3. As noted by Böttcher [97, Theorem 2.1 remains true in the case where $\varphi(z)$ is an invertible $N \times N$ matrix, provided the exponent $n+\#(\varphi)$ is replaced in (2.7), (2.8) by $N n+\#(\operatorname{det} \varphi)$. The proof in the scalar case extends to $N \times N$ matrices, and we give no further details : the proof in 9 is different and uses Wiener-Hopf factorization directly.

For the proof of Theorem 2.1, we use the following basic properties of the determinant (see, e.g., [33]). If $A$ is a trace class operator on a Hilbert space $H,\|A\|_{1}=\left(\operatorname{tr} A^{*} A\right)^{1 / 2}$ denotes the trace norm.

Lemma 2.4. (i). If $A_{n}$ is a trace class operator for each $n$ and $A_{n} \rightarrow A$ in trace norm, then $A$ is a trace class operator and $\operatorname{det}\left(1+A_{n}\right) \rightarrow \operatorname{det}(1+A)$ as $n \rightarrow \infty$.

(ii). If $A$ is a trace class operator, and $B_{n}$ and $C_{n}$ are bounded operators such that $\left(B_{n}\right)^{*}$ and $C_{n}$ converge strongly to $B^{*}$ and $C$ respectively, then $\operatorname{det}\left(1+C_{n} A B_{n}\right) \rightarrow \operatorname{det}(1+C A B)$ as $n \rightarrow \infty$.

(iii). If $A B$ and $B A$ are trace class operators, then $\operatorname{det}(1+A B)=\operatorname{det}(1+B A)$.

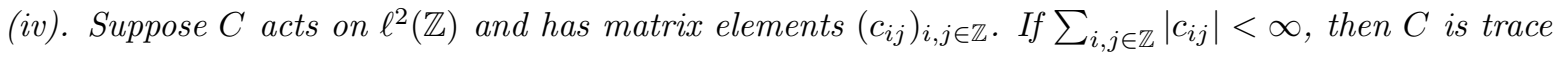
class and $\|C\|_{1} \leq \sum_{i, j \in \mathbb{Z}}\left|c_{i j}\right|$.

Proof of Theorem 2.1. Define the projection operators on the circle

$$
\left(P_{n} f\right)(z)=\sum_{j \geq n} f_{j} z^{j}, \quad n \in \mathbb{Z}
$$

and

$$
\begin{aligned}
& \left(Q_{n} f\right)(z)=\sum_{0 \leq j<n} f_{j} z^{j}, \quad n>0, \\
& \left(Q_{n} f\right)(z)=-\sum_{n \leq j<0} f_{j} z^{j}, \quad n<0,
\end{aligned}
$$

with $\left(Q_{0} f\right)(z)=0$. Thus in particular, we have $P_{n}=P_{0}-Q_{n}$. Let $M_{g}$ denote the multiplication operator

$$
\left(M_{g} f\right)(z)=g(z) f(z) .
$$


Direct calculation shows that

$$
\begin{aligned}
& \mathrm{K}_{\mathrm{n}}=-P_{0}+M_{\varphi} P_{n} M_{\varphi^{-1}}=\left(1-P_{0}\right)-M_{\varphi}\left(1-P_{n}\right) M_{\varphi^{-1}}, \\
& \mathrm{~S}_{\mathrm{n}}=P_{n} M_{\varphi^{-1}}\left(1-P_{0}\right) M_{\varphi} P_{n}, \\
& \mathrm{R}_{\mathrm{n}}=\left(1-P_{n}\right) M_{\varphi^{-1}} P_{0} M_{\varphi}\left(1-P_{n}\right) .
\end{aligned}
$$

First, we show that $\mathrm{K}_{\mathrm{n}}, \mathrm{S}_{\mathrm{n}}$ and $\mathrm{R}_{\mathrm{n}}$ are trace class. Indeed $\mathrm{K}_{\mathrm{n}}=-Q_{n}-H M_{\varphi^{-1}}$, where $H=\left[P_{n}, M_{\varphi}\right]$. $H$ acts on the basis $\left\{z^{l}\right\}_{l \in \mathbb{Z}}$ for $L^{2}(\Sigma, d w)$, as follows : $H z^{k}=\sum_{l} H_{l k} z^{l}$. We find

$$
H_{l k}=\varphi_{l-k}\left(\chi_{l \geq n}-\chi_{k \geq n}\right), \quad l, k \in \mathbb{Z},
$$

where $\chi_{\cdot \geq n}$ denotes the characteristic function of the set $\{k \geq n\}$. But $\sum_{l, k}\left|H_{l k}\right| \leq \sum_{j}\left|j \varphi_{j}\right|<\infty$, and hence by Lemma 2.4 (iv), we have the trace norm estimate

$$
\left\|\mathrm{K}_{\mathrm{n}}\right\|_{1} \leq n+\left(\sum_{j}\left|j \varphi_{j}\right|\right)\left\|\varphi^{-1}\right\|_{L^{\infty}} .
$$

Now write $\mathrm{S}_{\mathrm{n}}=A B$ where $A: \ell^{2}(\{1,2, \cdots\}) \rightarrow \ell^{2}(\{n, n+1, \cdots\})$ and $B: \ell^{2}(\{n, n+1, \cdots\}) \rightarrow$ $\ell^{2}(\{1,2, \cdots\})$ with matrix elements

$$
\begin{array}{ll}
A_{i k}=\left(\varphi^{-1}\right)_{i+k}, & i \geq n, k \geq 1, \\
B_{k j}=\varphi_{-k-j}, & k \geq 1, j \geq n .
\end{array}
$$

Write

$$
A=\chi_{n}^{+} \Phi^{-1} \chi_{-1}^{-} R
$$

where $(R f)_{j}=f_{-j}, \Phi^{-1}$ denotes convolution on $\ell^{2}(\mathbb{Z})$ by $\left\{\varphi_{j}^{-1}\right\}$,

$$
\left(\Phi^{-1} h\right)_{j}=\sum_{l \in \mathbb{Z}}\left(\varphi^{-1}\right)_{j-l} h_{l}
$$

and $\chi_{n}^{+}, \chi_{-1}^{-}$are the projections onto $\{k \geq n\}$ and $\{k \leq-1\}$ respectively. From (2.16), it is clear that $A$ is bounded from $\ell^{2}(\{1,2, \cdots\}) \rightarrow \ell^{2}(\{n, n+1, \cdots\})$ with norm estimate

$$
\|A\| \leq\left\|\varphi^{-1}\right\|_{L^{\infty}}
$$

On the other hand, a similar calculation to (2.14) shows that $B$ is trace class from $\ell^{2}(\{n, n+1, \cdots\}) \rightarrow$ $\ell^{2}(\{1,2, \cdots\})$ and

$$
\|B\|_{1} \leq \sum_{l \geq n+1}\left|l \varphi_{-l}\right| \leq \sum_{l}\left|l \varphi_{l}\right|
$$

which implies

$$
\left\|\mathrm{S}_{\mathrm{n}}\right\|_{1} \leq\left(\sum_{l \geq n+1}\left|(l+|n|) \varphi_{-l}\right|\right)\left\|\varphi^{-1}\right\|_{L^{\infty}} .
$$


Similarly, we have

$$
\left\|\mathrm{R}_{\mathrm{n}}\right\|_{1} \leq\left(\sum_{l \leq n}\left|(l+|n|) \varphi_{-l}\right|\right)\left\|\varphi^{-1}\right\|_{L^{\infty}} .
$$

Thus $\mathrm{K}_{\mathrm{n}}, \mathrm{S}_{\mathrm{n}}$ and $\mathrm{R}_{\mathrm{n}}$ are trace class. Moreover, if we set $\varphi_{J}:=\sum_{|j| \leq J} \varphi_{j} z^{j}, J \geq 0$, then from (the proofs of) (2.14), (2.20) and (2.21), it is clear that as $J \rightarrow \infty, \mathrm{K}_{\mathrm{n}}\left(\varphi_{J}\right) \rightarrow \mathrm{K}_{\mathrm{n}}(\varphi), \mathrm{S}_{\mathrm{n}}\left(\varphi_{J}\right) \rightarrow \mathrm{S}_{\mathrm{n}}(\varphi)$, $\mathrm{R}_{\mathrm{n}}\left(\varphi_{J}\right) \rightarrow \mathrm{R}_{\mathrm{n}}(\varphi)$ in trace norm, and hence the Fredholm determinants converge to the corresponding determinants. Also for $J$ sufficiently large, the winding number of $\varphi_{J}$ is the same as the winding number of $\varphi$, and so we see that to prove (2.7), it is enough to consider $\varphi$ 's which are non-zero and analytic in a neighborhood of $\Sigma$. Henceforth we will assume that $\varphi$ is analytic : this analyticity assumption is not necessary and is used only to give a particularly simple proof of Lemma 2.5 below.

In the below, we only present the proof of (2.7). The proof of (2.8) is similar. Formally, we proceed as follows. Suppose $P_{n}$ is finite rank so that $P_{0}=Q_{n}+P_{n}$ is also finite rank. We have

$$
\operatorname{det}\left(1+s P_{0}-s M_{\varphi} P_{n} M_{\varphi^{-1}}\right)=\operatorname{det}\left(1+s P_{0}\right) \operatorname{det}\left(1-\frac{s}{1+s P_{0}} M_{\varphi} P_{n} M_{\varphi^{-1}}\right)
$$

Using $P_{0}=Q_{n}+P_{n}$ and $\frac{1}{1+s P_{0}}=1-\frac{s}{1+s} P_{0}$, the right-hand-side reduces to

$$
\begin{cases}\operatorname{det}\left(1+s Q_{n}\right) \operatorname{det}\left(1+s P_{n}\right) \operatorname{det}\left(1-s\left(1-\frac{s}{1+s} P_{0}\right) M_{\varphi} P_{n} M_{\varphi^{-1}}\right), & n \geq 0, \\ \left(\operatorname{det}\left(1-s Q_{n}\right)\right)^{-1} \operatorname{det}\left(1+s P_{n}\right) \operatorname{det}\left(1-s\left(1-\frac{s}{1+s} P_{0}\right) M_{\varphi} P_{n} M_{\varphi^{-1}}\right), & n<0 .\end{cases}
$$

The first term in both cases is equal to $(1+s)^{n}$. Using Lemma 2.4 [iii] and $P_{n}=P_{n}^{2}$ for the last determinant, (2.23) becomes

$$
\begin{aligned}
(1 & +s)^{n} \operatorname{det}\left(1+s P_{n}\right) \operatorname{det}\left(1-s P_{n} M_{\varphi^{-1}}\left(1-\frac{s}{1+s} P_{0}\right) M_{\varphi} P_{n}\right) \\
& =(1+s)^{n} \operatorname{det}\left(\left(1+s P_{n}\right)-s(1+s) P_{n} M_{\varphi^{-1}}\left(1-\frac{s}{1+s} P_{0}\right) M_{\varphi} P_{n}\right) \\
& =(1+s)^{n} \operatorname{det}\left(1-s^{2} \mathrm{~S}_{\mathrm{n}}\right),
\end{aligned}
$$

which is the desired result, up to the winding number $\#(\varphi)$. For the case in hand, however, $P_{0}$ is not a trace class operator and the above "proof" breaks down. We circumvent the difficulty by approximating the operator $K_{\mathrm{n}}$ by finite rank operators, and the missing factor \# $(\varphi)$ will appear along the way.

Let $T_{N}$ be the projection

$$
\left(T_{N} f\right)(z)=\sum_{|j| \leq N} f_{j} z^{j}, \quad N \geq 1
$$

Note that $T_{N}$ is a trace class operator since it has finite rank. Clearly $T_{N}, T_{N}^{*} \rightarrow 1$ strongly, and hence by Lemma 2.4 (ii),

$$
\operatorname{det}\left(1-s \mathrm{~K}_{\mathrm{n}}\right)=\lim _{N \rightarrow \infty} \operatorname{det}\left(1+s\left(P_{0}-M_{\varphi} P_{n} M_{\varphi^{-1}}\right) T_{N}\right)
$$

Now since $P_{k} T_{N}$ is trace class, proceeding as above in (2.22)-(2.24), we have for $N \geq|n|$, $\operatorname{det}\left(1+s\left(P_{0}-M_{\varphi} P_{n} M_{\varphi^{-1}}\right) T_{N}\right)=(1+s)^{n} \operatorname{det}\left(\left(1+s P_{n} T_{N}\right)\left(1-s P_{n} M_{\varphi^{-1}} T_{N}\left(1-\frac{s}{1+s} P_{0} T_{N}\right) M_{\varphi} P_{n}\right)\right)$. 
Thus we have

$$
\operatorname{det}\left(1-s \mathrm{~K}_{\mathrm{n}}\right)=(1+s)^{n} \lim _{N \rightarrow \infty} \operatorname{det}\left(1+X_{N}+Y_{N}\right)
$$

where for $N \geq n$,

$$
\begin{aligned}
X_{N} & =s \frac{1+s T_{N}}{1+s} P_{n}\left(T_{N}-M_{\varphi^{-1}} T_{N} M_{\varphi}\right) P_{n} \\
Y_{N} & =-s^{2} \frac{1+s T_{N}}{1+s} P_{n} M_{\varphi^{-1}} T_{N}\left(1-P_{0}\right) M_{\varphi} P_{n} .
\end{aligned}
$$

We observe that

(i). $X_{N}$ and $Y_{N}$ are trace class.

(ii). $X_{N}^{*} \rightarrow 0$ strongly as $N \rightarrow \infty$.

(iii). $Y_{N} \rightarrow-s^{2} \mathrm{~S}_{\mathrm{n}}$ in trace norm as $N \rightarrow \infty$.

(iv). $\left(1+X_{N}\right)^{-1}$ and $\left(1+Y_{N}\right)^{-1}$ are uniformly bounded in operator norm as $N \rightarrow \infty$ when $s$ is small enough.

The third property follows using Lemma 2.4 (ii) as $\left(1-P_{0}\right) M_{\varphi} P_{n}$ is trace class and $T_{N} \rightarrow 1$ strongly.

For a moment, we assume that $s$ is small so that (iv) is satisfied. Now we rewrite the right hand side of (2.28) as

$$
\operatorname{det}\left(1+X_{N}+Y_{N}\right)=\operatorname{det}\left(1+Y_{N}\right) \operatorname{det}\left(1+X_{N}\right) \operatorname{det}\left(1-\frac{1}{1+X_{N}} \frac{1}{1+Y_{N}} Y_{N} X_{N}\right) .
$$

From the properties (i), (ii), (iv) above, we have $\frac{1}{1+X_{N}} \frac{1}{1+Y_{N}} Y_{N} X_{N} \rightarrow 0$ in trace norm. Using the property (iii), we now have

$$
\operatorname{det}\left(1-s \mathrm{~K}_{\mathrm{n}}\right)=(1+s)^{n} \operatorname{det}\left(1-s^{2} \mathrm{~S}_{\mathrm{n}}\right) \lim _{N \rightarrow \infty} \operatorname{det}\left(1+X_{N}\right)
$$

Rewrite $X_{N}$ as

$$
\begin{aligned}
X_{N} & =s \frac{1+s T_{N}}{1+s} P_{n}\left(M_{\varphi^{-1}}\left(1-T_{N}\right) M_{\varphi}-\left(1-T_{N}\right)\right) P_{n} \\
& =s \frac{1+s T_{N}}{1+s} P_{n}\left(M_{\varphi^{-1}} P_{N+1} M_{\varphi}-P_{N+1}\right) P_{n}+s \frac{1+s T_{N}}{1+s} P_{n}\left(M_{\varphi^{-1}}\left(1-P_{-N}\right) M_{\varphi}-\left(1-P_{-N}\right)\right) P_{n} \\
& =: Z_{N}+W_{N} .
\end{aligned}
$$

Then as $N \geq|n|$,

$$
W_{N}=s \frac{1+s T_{N}}{1+s} P_{n} M_{\varphi^{-1}}\left(1-P_{-N}\right) M_{\varphi} P_{n}=s \frac{1+s T_{N}}{1+s} P_{n} M_{\varphi^{-1}}\left(1-P_{-N}\right)\left(1-P_{0}\right) M_{\varphi} P_{n},
$$

and hence $W_{N} \rightarrow 0$ in trace norm as $\left(1-P_{-N}\right) \rightarrow 0$ strongly and $\left(1-P_{0}\right) M_{\varphi} P_{n}$ is in trace class. Also $Z_{N} \rightarrow 0$ strongly, and $\left(1+Z_{N}\right)^{-1},\left(1+W_{N}\right)^{-1}$ are uniformly bounded as $N \rightarrow \infty$ for $s$ small enough. Thus by similar arguments leading to (2.32), we have

$$
\lim _{N \rightarrow \infty} \operatorname{det}\left(1+X_{N}\right)=\lim _{N \rightarrow \infty} \operatorname{det}\left(1+Z_{N}\right)
$$


Now by (2.12) and (2.1), we note that

$$
M_{\varphi^{-1}} P_{N+1} M_{\varphi}-P_{N+1}=\tilde{\mathrm{K}}_{\mathrm{N}+1}+Q_{N+1}=A_{N+1} \tilde{\mathrm{K}}_{0} A_{N+1}^{-1},
$$

where $\tilde{\mathrm{K}}_{\mathrm{N}+1}$ is $\mathrm{K}_{\mathrm{N}+1}$ with $\varphi$ replaced by $\varphi^{-1}$, and $A_{N+1}$ is the operator of multiplication by $z^{N+1}$. Thus we have

$$
\begin{aligned}
\operatorname{det}\left(1+Z_{N}\right) & =\operatorname{det}\left(1+s \frac{1+s T_{N}}{1+s} P_{n} A_{N+1} \tilde{\mathrm{K}}_{0} A_{N+1}^{-1} P_{n}\right) \\
& =\operatorname{det}\left(1+s \tilde{\mathrm{K}}_{0} A_{N+1}^{-1} P_{n} \frac{1+s T_{N}}{1+s} P_{n} A_{N+1}\right),
\end{aligned}
$$

by Lemma 2.4 (iii). Since $P_{k} A_{N+1}=A_{N+1} P_{k-N-1}$, and $T_{N}=P_{-N}-P_{N+1}$, we have

$$
A_{N+1}^{-1} P_{n} \frac{1+s T_{N}}{1+s} P_{n} A_{N+1}=\frac{1}{1+s}\left((1+s) P_{n-N-1}-s P_{0}\right) \rightarrow \frac{1+s-s P_{0}}{1+s}=: D(s)
$$

strongly. Since $\tilde{\mathrm{K}}_{0}$ is a trace class, we obtain

$$
\lim _{N \rightarrow \infty} \operatorname{det}\left(1+Z_{N}\right)=\operatorname{det}\left(1+s \tilde{\mathrm{K}}_{0} D(s)\right)
$$

Therefore from (2.32) and (2.35),

$$
\operatorname{det}\left(1-s \mathrm{~K}_{\mathrm{n}}\right)=(1+s)^{n} \operatorname{det}\left(1-s^{2} S_{n}\right) \operatorname{det}\left(1+s \tilde{\mathrm{K}}_{0} D(s)\right)
$$

Since $\operatorname{det}\left(1+s \tilde{\mathrm{K}}_{0} D(s)\right)$ does not depend on $n$, we obtain the value of this determinant by letting $n \rightarrow \infty$ in both sides of (2.40). But by Lemma 2.5 below, for small $s,(1+s)^{-n} \operatorname{det}\left(1-s \mathrm{~K}_{\mathrm{n}}\right) \rightarrow$ $(1+s)^{\#(\varphi)}$ as $n \rightarrow \infty$. On the other hand, from (2.20), $\operatorname{det}\left(1-s^{2} S_{n}\right)$ converges to 1 . Therefore $\operatorname{det}\left(1+s \tilde{\mathrm{K}}_{0} D(s)\right)=(1+s)^{\#(\varphi)}$, and we obtain, for small $s$,

$$
\operatorname{det}\left(1-s \mathrm{~K}_{\mathrm{n}}\right)=(1+s)^{n+\#(\varphi)} \operatorname{det}\left(1-s^{2} S_{n}\right),
$$

as desired. The result for all $s$ now follows by analytic continuation.

Observe from (2.1), 2.12) that $\left\|\mathrm{K}_{\mathrm{n}}\right\| \leq 1+\|\varphi\|_{\infty}\left\|\varphi^{-1}\right\|_{\infty}=: s_{0}$. (A different estimate (see Appendix [4]) shows that $\left\|K_{n}\right\| \leq \max \left(\|\varphi\|_{\infty},\left\|\varphi^{-1}\right\|_{\infty}\right)$.) Then we have the following result.

Lemma 2.5. For a function $\varphi$ which is analytic and non-zero in a neighborhood of the unit circle

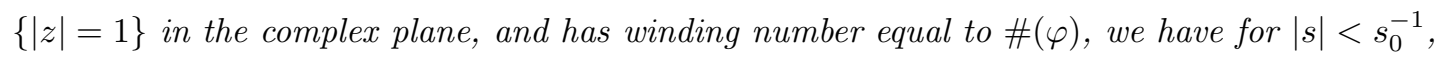

$$
\begin{aligned}
& \lim _{n \rightarrow \infty}(1+s)^{-n} \operatorname{det}\left(1-s \mathrm{~K}_{\mathrm{n}}\right)=(1+s)^{\#(\varphi)}, \\
& \lim _{n \rightarrow-\infty}(1-s)^{n} \operatorname{det}\left(1-s \mathrm{~K}_{\mathrm{n}}\right)=(1-s)^{-\#(\varphi)},
\end{aligned}
$$

for some $s_{0}>0$. 
Proof. In Lemma 5 of 仼, we obtained the result 2.42) for $\varphi(z)=e^{\sqrt{\lambda(}\left(z-z^{-1}\right)}$ and $s=\sqrt{t}, 0 \leq t \leq 1$. For general analytic $\varphi$ and $s$ small, the proof remains the same until equation (50). The second component in the asymptotics of $F^{\prime}$ is now $-\frac{\varphi^{\prime}}{1+s^{\prime}}$, and hence $(51)$ is changed to

$$
\begin{aligned}
\log \operatorname{det}\left(1-s \mathrm{~K}_{\mathrm{n}}\right) & =-\int_{0}^{s} \frac{d s^{\prime}}{2 \pi i\left(1+s^{\prime}\right)} \int_{|z|=1}\left[-n z^{-1}-\frac{\varphi^{\prime}(z)}{\varphi(z)}\right] d z+O\left(e^{-c n}\right) \\
& =(n+\#(\varphi)) \log (1+s)+O\left(e^{-c n}\right) .
\end{aligned}
$$

The calculation for (2.43) is similar and we skip the details.

Remark 2.6. Lemma 2.5 does not require $\varphi$ to be analytic. However, in this case, the proof is particularly simple, and can be quoted directly from [A] as above.

Remark 2.7. The fact that $\operatorname{det}\left(1+s \tilde{\mathrm{K}}_{0} D(s)\right)=(1+s)^{\#(\varphi)}$ is rather remarkable. It is an instructive exercise to check this identity directly when $\varphi$ is simple, say $\varphi=z^{k}$ or $\varphi=(1+a z)\left(1+b z^{-1}\right),|a|,|b|<1$.

Remark 2.8. By (2.7), we see that if $n+\#(\varphi)>0, \operatorname{det}\left(1-s \mathrm{~K}_{\mathrm{n}}\right)$ has a root at $s=-1$ of order at least $n+\#(\varphi)$. In particular, $\mathrm{K}_{\mathrm{n}}$ has eigenvalue -1 . Moreover, if $\mathrm{K}_{\mathrm{n}}$ is self-adjoint (which is true by (2.12) whenever $|\varphi|=1$, e.g., $\varphi=e^{\sqrt{\lambda}\left(z-z^{-1}\right)}$ as in [3, 团), then $\mathrm{K}_{\mathrm{n}}$ has an eigenspace of dimension at least $n+\#(\varphi)$ corresponding to the eigenvalue -1 . It is also clear from (2.7) that if $s \neq-1$ is a root of $\operatorname{det}\left(1-s \mathrm{~K}_{\mathrm{n}}\right)$, then so is $-s$. On the other hand, if $n+\#(\varphi)<0$, then clearly $\operatorname{det}\left(1-s^{2} \mathrm{~S}_{\mathrm{n}}\right)$ has a root at $s= \pm 1$, etc. In the self-adjoint case, when $|\varphi|=1$, we see from (2.12) that $\mathrm{S}_{\mathrm{n}}$ is positive definite with norm $\leq 1$. We will use this fact in Section 5 .

Remark 2.9. Define the operator $A$ acting on $\ell(\mathbb{Z})$ by

$$
A=M_{\varphi^{-1}} P_{0} M_{\varphi}
$$

Since $\operatorname{det}\left(1-s \mathrm{~K}_{\mathrm{n}}\right)=\operatorname{det}\left(1-s M_{\varphi^{-1}} \mathrm{~K}_{\mathrm{n}} M_{\varphi}\right)$, using (2.12), the above theorem can be rephrased as

$$
\begin{aligned}
\operatorname{det}\left(1-s\left(P_{n}-A\right)\right) & =(1+s)^{n+\#(\varphi)} \operatorname{det}\left(1-s^{2} P_{n}(1-A) P_{n}\right) \\
& =(1-s)^{-n-\#(\varphi)} \operatorname{det}\left(1-s^{2}\left(1-P_{n}\right) A\left(1-P_{n}\right)\right) .
\end{aligned}
$$

These are the identities (8.55), (8.56) in [3] for a certain subclass of $\varphi$ 's with zero winding, $\#(\varphi)=0$.

The following Corollary will be used in the analysis of (S3) in Section 7 below.

Corollary 2.10. Let $\varphi(z)$ be as in Theorem 2.1. Define $\mathrm{K}_{\mathrm{n}}^{(\mathrm{m})}, \mathrm{S}^{(\mathrm{m})}$ and $\mathrm{R}^{(\mathrm{m})}$ to be the operators analogous to $\mathrm{K}_{\mathrm{n}}, \mathrm{S}^{(\mathrm{m})}$ and $\mathrm{R}^{(\mathrm{m})}$ with the matrix elements given by

$$
\mathrm{K}_{\mathrm{n}}^{(\mathrm{m})}(z, w)=\frac{1-z^{n} \varphi\left(z^{m}\right) w^{-n} \varphi\left(w^{m}\right)^{-1}}{2 \pi i(z-w)}
$$


and

$$
\begin{aligned}
\mathrm{S}^{(\mathrm{m})}(i, j) & =\sum_{k \geq 1}\left(\varphi^{-1}\right)_{(i+k) / m} \varphi_{(-j-k) / m} \\
\mathrm{R}^{(\mathrm{m})}(i, j) & =\sum_{k \leq 0}\left(\varphi^{-1}\right)_{(i+k) / m} \varphi_{(-j-k) / m}
\end{aligned}
$$

where $\varphi_{a}=\left(\varphi^{-1}\right)_{a}=0$ if $a \neq \mathbb{Z}$. Set $\mathrm{S}_{\mathrm{n}}^{(\mathrm{m})}=\chi_{[n, \infty)} \mathrm{S}^{(\mathrm{m})}$ and $\mathrm{R}_{\mathrm{n}}^{(\mathrm{m})}=\chi_{(-\infty, n-1]} \mathrm{R}^{(\mathrm{m})}$. Then we have

$$
\begin{aligned}
\operatorname{det}\left(1-s \mathrm{~K}_{\mathrm{n}}^{(\mathrm{m})}\right) & =(1+s)^{n+\#(\varphi)} \operatorname{det}\left(1-s^{2} \mathrm{~S}_{\mathrm{n}}^{(\mathrm{m})}\right), \quad s \neq-1, \\
& =(1-s)^{-n-\#(\varphi)} \operatorname{det}\left(1-s^{2} \mathrm{R}_{\mathrm{n}}^{(\mathrm{m})}\right), \quad s \neq 1 .
\end{aligned}
$$

Remark 2.11. Observe that $\mathrm{S}_{\mathrm{n}}^{(\mathrm{m})}$ has the block structure

$$
\left(\begin{array}{ccc}
\mathrm{S}_{\mathrm{n}}^{(\mathrm{m})}(m i, m j) & \cdots & \mathrm{S}_{\mathrm{n}}^{(\mathrm{m})}(m i, m j+m-1) \\
\cdots & \cdots & \cdots \\
\mathrm{S}_{\mathrm{n}}^{(\mathrm{m})}(m i+m-1, m j) & \cdots & \mathrm{S}_{\mathrm{n}}^{(\mathrm{m})}(m i+m-1, m j+m-1)
\end{array}\right)=\left(\begin{array}{cccc}
\mathrm{S}_{\mathrm{n}}(i, j) & 0 & \cdots & 0 \\
0 & \mathrm{~S}_{\mathrm{n}}(i, j) & \cdots & 0 \\
& \cdots & & \\
0 & 0 & \cdots & \mathrm{S}_{\mathrm{n}}(i, j)
\end{array}\right) .
$$

For the multi-interval case, we can generalize the argument in Theorem 2.1 to obtain the following result.

Theorem 2.12. Let $0=n_{0} \leq n_{1} \leq n_{2} \leq \cdots \leq n_{k} \leq n_{k+1}=\infty$ be integers, and let $s_{1}, \cdots, s_{k}$ be complex numbers satisfying $s_{k} \neq-1$ and $s_{k}-s_{j} \neq-1$. Also set $s_{0}=0$. We have

$$
\begin{aligned}
& \operatorname{det}\left(1-\sum_{j=1}^{k}\left(s_{j}-s_{j-1}\right) \mathrm{K}_{n_{j}}\right) \\
& \quad=\left(1+s_{k}\right) \#(\varphi)\left[\prod_{j=0}^{k-1}\left(1+s_{k}-s_{j}\right)^{n_{j+1}-n_{j}}\right] \operatorname{det}\left(1-\left(\sum_{j=1}^{k} \frac{s_{k} s_{j}}{1+s_{k}-s_{j}} \chi_{\left[n_{j}, n_{j+1}\right)}\right) \mathrm{S}\right)
\end{aligned}
$$

where $\#(\varphi)$ is again the winding number of $\varphi$.

Remark 2.13. As noted by Böttcher (河; ; ef. Remark 2.3 above), the formula (2.54) remains true in the $N \times N$ matrix case, provided we replace $n_{j+1}-n_{j}$ by $N\left(n_{j+1}-n_{j}\right), 0 \leq j \leq k-1$, and \# $(\varphi)$ by $\#(\operatorname{det} \varphi)$. Again the proof in the scalar case extends to the matrix case, and we provide no details.

Proof. The formal procedure (without considering the winding number) is as follows. For $j=0, \cdots, k-$ 1, let $R_{j}$ be the projection operator on $\left\{n_{j}, \cdots, n_{j+1}-1\right\}$, and let $R_{k}$ be the projection operator on $\left\{n_{k}, n_{k}+1 \cdots\right\}$. Since we have from (2.12)

$$
\mathrm{K}_{n_{j}}=-\sum_{l=0}^{k} R_{l}+M_{\varphi}\left(\sum_{l=j}^{k} R_{l}\right) M_{\varphi^{-1}}, \quad j=1, \cdots, k,
$$


the determinant on the left-hand-side in (2.54), denoted by $(*)$, is equal to

$$
(*)=\operatorname{det}\left(1+s_{k} \sum_{j=0}^{k} R_{j}-M_{\varphi}\left(\sum_{j=1}^{k} s_{j} R_{j}\right) M_{\varphi^{-1}}\right) .
$$

First we pull out the term $1+s_{k} \sum_{j=0}^{k} R_{j}$, then use Lemma 2.4 (iii) to obtain

$$
\begin{aligned}
(*) & =\operatorname{det}\left(1+s_{k} \sum_{j=0}^{k} R_{j}\right) \operatorname{det}\left(1-\frac{1}{1+s_{k} \sum_{j=0}^{k} R_{j}} M_{\varphi}\left(\sum_{j=1}^{k} s_{j} R_{j}\right) M_{\varphi^{-1}}\right) \\
& =\operatorname{det}\left(1+s_{k} \sum_{j=0}^{k} R_{j}\right) \operatorname{det}\left(1-\left(\sum_{j=1}^{k} s_{j} R_{j}\right) M_{\varphi^{-1}} \frac{1}{1+s_{k} \sum_{j=0}^{k} R_{j}} M_{\varphi}\right) .
\end{aligned}
$$

Now note that (recall $s_{0}=0$ )

$$
\begin{aligned}
\operatorname{det}\left(1+s_{k} \sum_{j=0}^{k} R_{j}\right) & =\operatorname{det}\left(1+\sum_{j=0}^{k-1}\left(s_{k}-s_{j}\right) R_{j}+\sum_{j=1}^{k} s_{j} R_{j}\right) \\
& =\operatorname{det}\left(1+\sum_{j=0}^{k-1}\left(s_{k}-s_{j}\right) R_{j}\right) \operatorname{det}\left(1+\frac{1}{1+\sum_{j=0}^{k-1}\left(s_{k}-s_{j}\right) R_{j}} \sum_{j=1}^{k} s_{j} R_{j}\right) \\
& =\left[\prod_{j=0}^{k-1}\left(1+s_{k}-s_{j}\right)^{n_{j+1}-n_{j}}\right] \operatorname{det}\left(1+\sum_{j=1}^{k} \frac{s_{j}}{1+s_{k}-s_{j}} R_{j}\right) .
\end{aligned}
$$

Using (2.58) and then multiplying two determinants, we have

$$
\begin{aligned}
(*)= & {\left[\prod_{j=0}^{k-1}\left(1+s_{k}-s_{j}\right)^{n_{j+1}-n_{j}}\right] } \\
& \quad \times \operatorname{det}\left(1+\sum_{j=1}^{k} \frac{s_{j}}{1+s_{k}-s_{j}} R_{j}-\left(1+s_{k}\right)\left(\sum_{j=1}^{k} \frac{s_{j}}{1+s_{k}-s_{j}} R_{j}\right) M_{\varphi^{-1}} \frac{1}{1+s_{k} \sum_{j=0}^{k} R_{j}} M_{\varphi}\right) .
\end{aligned}
$$

Finally, using

$$
\frac{1}{1+s_{k} \sum_{j=0}^{k} R_{j}}=\frac{1+s_{k}\left(1-\sum_{j=0}^{k} R_{j}\right)}{1+s_{k}}
$$

in the determinant on the right-hand-side of (2.59), we obtain

$$
(*)=\left[\prod_{j=0}^{k-1}\left(1+s_{k}-s_{j}\right)^{n_{j+1}-n_{j}}\right] \operatorname{det}\left(1-\sum_{j=0}^{k} \frac{s_{k} s_{j}}{1+s_{k}-s_{j}} R_{j} M_{\varphi^{-1}}\left(1-\sum_{j=0}^{k} R_{j}\right) M_{\varphi}\right),
$$

which is precisely (2.54) from (2.12).

The rigorous proof is also similar to the proof of Theorem 2.1. Let $T_{N}$ be the projection on $|j| \leq N$ as in (2.25). We take $N$ large so that $N>n_{k}$. The analogue of (2.28) is now

$$
(*)=\left[\prod_{j=0}^{k-1}\left(1+s_{k}-s_{j}\right)^{n_{j+1}-n_{j}}\right] \lim _{N \rightarrow \infty} \operatorname{det}\left(1+X_{N}+Y_{N}\right),
$$


where

$$
\begin{aligned}
X_{N} & =\left(\sum_{j=1}^{k} \frac{s_{j}}{1+s_{k}-s_{j}} \frac{1+s_{k}-s_{j}\left(1-T_{N}\right)}{1+s_{k}} R_{j}\right)\left(T_{N}-M_{\varphi^{-1}} T_{N} M_{\varphi}\right) \\
Y_{N} & =-\left(\sum_{j=1}^{k} \frac{s_{k} s_{j}}{1+s_{k}-s_{j}} \frac{1+s_{k}-s_{j}\left(1-T_{N}\right)}{1+s_{k}} R_{j}\right) M_{\varphi^{-1}} T_{N}\left(1-\sum_{j=0}^{k} s_{j} R_{j}\right) M_{\varphi}
\end{aligned}
$$

which becomes, by the same argument leading to (2.35),

$$
(*)=\left[\prod_{j=0}^{k-1}\left(1+s_{k}-s_{j}\right)^{n_{j+1}-n_{j}}\right] \operatorname{det}\left(1-\left(\sum_{j=1}^{k} \frac{s_{k} s_{j}}{1+s_{k}-s_{j}} \chi_{\left[n_{j}, n_{j+1}\right)}\right) \mathrm{S}\right) \lim _{N \rightarrow \infty} \operatorname{det}\left(1+Z_{N}\right),
$$

with $Z_{N}$ in (2.33) where $s$ is replaced by $s_{k}$, This then leads to the desired result as in the single interval case.

\section{Convergence of moments}

In this section, we prove the convergence of moments for arbitrary (scaled) rows, $\xi_{j}$, of a random young diagram under the Plancherel measure, mentioned in the Introduction. The tail estimates used in the proof of Theorem 3.1 are given in Section 1 below.

Let $\mathbb{N}_{0}:=\mathbb{N} \cup\{0\}$.

Theorem 3.1. For any fixed $k \in \mathbb{N}$, and for any $a_{j} \in \mathbb{N}_{0}, 1 \leq j \leq k$, we have as $N \rightarrow \infty$

$$
\mathbb{E}_{N}^{\mathrm{Plan}}\left(\xi_{1}^{a_{1}} \cdots \xi_{k}^{a_{k}}\right) \rightarrow \mathbb{E}\left(x_{1}^{a_{1}} \cdots x_{k}^{a_{k}}\right)
$$

where $\mathbb{E}_{N}^{\text {Plan }}$ denotes the expectation with respect to the Plancherel measure on $Y_{N}$, and $\mathbb{E}$ denotes the expectation with respect to the limiting distribution function $F$ in (11.6), (1.7).

Remark 3.2. It will be clear from the proof below that the following stronger convergence result is also true : Let $h_{j}(x), j=1, \cdots, k$ be continuous functions on $\mathbb{R}$ satisfying $\left|h_{j}(x)\right| \leq C_{1} e^{c_{2}|x|^{3 / 2-\epsilon}}$ for some $\epsilon>0$. Then for any $k$, as $N \rightarrow \infty$,

$$
\mathbb{E}_{N}^{\text {Plan }}\left(h_{1}\left(\xi_{1}\right) \cdots h_{k}\left(\xi_{k}\right)\right) \rightarrow \mathbb{E}\left(h_{1}\left(x_{1}\right) \cdots h_{k}\left(x_{k}\right)\right) .
$$

Proof. We have

$$
\mathbb{E}_{N}^{\mathrm{Plan}}\left(\xi_{1}^{a_{1}} \cdots \xi_{k}^{a_{k}}\right)=\int_{x_{1} \geq \cdots \geq x_{k}} \prod_{j=1}^{k} x_{j}^{a_{j}} d \mathbb{P}_{N}^{\mathrm{Plan}}\left(\xi_{1} \leq x_{1}, \cdots, \xi_{k} \leq x_{k}\right)
$$

since $\lambda_{1} \geq \lambda_{2} \cdots$. Fix a number $T>2$. We split the integral into two pieces :

$$
\begin{aligned}
& \text { (a) } \max _{1 \leq j \leq k}\left|x_{j}\right| \leq T \\
& \text { (b) } \max _{1 \leq j \leq k}\left|x_{j}\right|>T .
\end{aligned}
$$


In the first part (a), using a standard argument and the convergence in distribution (1.7) above, the limit becomes as $N \rightarrow \infty$

$$
\int_{\substack{x_{1} \geq \cdots \geq x_{k} \\ \max \left|x_{j}\right| \leq T}} \prod_{j=1}^{k} x_{j}^{a_{j}} d F\left(x_{1}, \cdots, x_{k}\right) .
$$

For the second part (b), the region is a union of two (not necessarily disjoint) pieces :

$$
\begin{aligned}
& \text { (i) } \max _{j}\left|x_{j}\right|=\left|x_{1}\right| \\
& \text { (ii) } \max _{j}\left|x_{j}\right|=\left|x_{k}\right| .
\end{aligned}
$$

Note that since $x_{1} \geq \cdots \geq x_{k}, \max _{j}\left|x_{j}\right|$ is either $\left|x_{1}\right|$ or $\left|x_{k}\right|$. Over region (i),

$$
\begin{aligned}
\int_{(i)} \prod_{j=1}^{k}\left|x_{j}\right|^{a_{j}} d \mathbb{P}_{N}^{\text {Plan }}\left(\xi_{1} \leq x_{1}, \cdots, \xi_{k} \leq x_{k}\right) & \leq \int_{(i)}\left|x_{1}\right|^{a_{1}+\cdots+a_{k}} d \mathbb{P}_{N}^{\text {Plan }}\left(\xi_{1} \leq x_{1}, \cdots, \xi_{k} \leq x_{k}\right) \\
& \leq \int_{(-\infty,-T) \cup(T, \infty)}\left|x_{1}\right|^{a_{1}+\cdots+a_{k}} d \mathbb{P}_{N}^{\text {Plan }}\left(\xi_{1} \leq x_{1}\right) \\
& =\mathbb{E}_{N}^{\text {Plan }}\left(\left|\xi_{1}\right|^{a_{1}+\cdots+a_{k}}\left(\chi_{\xi_{1}<-T}+\chi_{\xi_{1}>T}\right)\right) .
\end{aligned}
$$

Similarly,

$$
\int_{(i i)} \prod_{j=1}^{k}\left|x_{j}\right|^{a_{j}} d \mathbb{P}_{N}^{\mathrm{Plan}}\left(\xi_{1} \leq x_{1}, \cdots, \xi_{k} \leq x_{k}\right) \leq \mathbb{E}_{N}^{\mathrm{Plan}}\left(\left|\xi_{k}\right|^{a_{1}+\cdots+a_{k}}\left(\chi_{\xi_{k}<-T}+\chi_{\xi_{k}>T}\right)\right) .
$$

Now from the tail estimates in Proposition 4.3 below, the moment (3.3) as $N \rightarrow \infty$ is equal to (3.6) plus a term which can be made arbitrarily small if we take $T$ large enough. However, from Lemma 3.3 , for $T$ large, (3.6) is arbitrarily close to $\mathbb{E}\left(x_{1}^{a_{1}} \cdots x_{k}^{a_{k}}\right)$. Thus we have proved the theorem.

Lemma 3.3. For any $k \in \mathbb{N}$, and for any $a_{j} \in \mathbb{N}_{0}, 1 \leq j \leq k$,

$$
\mathbb{E}\left(\left|x_{1}\right|^{a_{1}} \cdots\left|x_{k}\right|^{a_{k}}\right)<\infty
$$

where $\mathbb{E}$ is the expectation with respect to the limiting distribution function $F$ in (1.6) and (1.7).

Proof. We need to show that

$$
\int_{x_{1} \geq \cdots \geq x_{k}} \prod_{j=1}^{k}\left|x_{j}\right|^{a_{j}} d F\left(x_{1}, \cdots, x_{k}\right)<\infty
$$

Fix $T>2$. We split the integral into two parts as in (3.4), (3.5): (a) $\max _{j}\left|x_{j}\right| \leq T$, and (b) $\max _{j}\left|x_{j}\right|>T$. In (a), the integral is finite. In (b), the argument yielding (3.9), (3.10) implies that

$$
\int_{(b)} \prod_{j=1}^{k}\left|x_{j}\right|^{a_{j}} d F\left(x_{1}, \cdots, x_{k}\right) \leq \mathbb{E}\left(\left|x_{1}\right|^{a_{1}+\cdots+a_{k}} \chi_{x_{1}>T}\right)+\mathbb{E}\left(\left|x_{k}\right|^{a_{1}+\cdots+a_{k}} \chi_{x_{k}<-T}\right) .
$$


(Note that the additional terms corresponding to $\mathbb{E}_{N}^{\text {Plan }}\left(\left|\xi_{1}\right|^{a_{1}+\cdots+a_{k}} \chi_{\xi_{1}<T}\right)$ in $(3.9)$ and $\mathbb{E}_{N}^{\text {Plan }}\left(\left|\xi_{k}\right|^{a_{1}+\cdots+a_{k}} \chi_{\xi_{k}>T}\right)$ in (3.10) are not necessary here as $d F$ is a smooth measure.) We will prove the finiteness of the last two expected values for $a=a_{1}+\cdots+a_{k}$.

First, we prove that $\mathbb{E}\left(x_{1}^{a} \chi_{x_{1}>T}\right)<\infty$ for any $a \in \mathbb{N}_{0}$. Note that by (1.7) and (4.8) below, for $x_{1}>T_{0}$,

$$
1-F\left(x_{1}\right)=\lim _{N \rightarrow \infty} 1-\mathbb{P}_{N}^{\mathrm{Plan}}\left(\xi_{1} \leq x_{1}\right)=\lim _{N \rightarrow \infty} \mathbb{E}_{N}^{\mathrm{Plan}}\left(\chi_{\xi>x_{1}}\right) \leq C e^{-c x_{1}^{3 / 2}}
$$

for some $C, c>0$. In particular, we have for any $a \in \mathbb{N}_{0}$,

$$
\lim _{x_{1} \rightarrow \infty} x_{1}^{a}\left(1-F\left(x_{1}\right)\right)=0 .
$$

Thus, integrating by parts,

$$
\int_{T}^{\infty} x_{1}^{a} d F\left(x_{1}\right)=T^{a}(1-F(T))+\int_{T}^{\infty} a x_{1}^{a-1}\left(1-F\left(x_{1}\right)\right) d x_{1} .
$$

Using (1.7), Fatou's lemma and (4.8), we have

$$
\begin{aligned}
\mathbb{E}\left(x_{1}^{a} \chi_{x_{1}>T}\right) & =\int_{T}^{\infty} x_{1}^{a} d F\left(x_{1}\right) \\
& =\lim _{N \rightarrow \infty} T^{a}\left(1-\mathbb{P}_{N}^{\text {Plan }}\left(\xi_{1} \leq T\right)\right)+\int_{T}^{\infty} \lim _{N \rightarrow \infty} a x_{1}^{a-1}\left(1-\mathbb{P}_{N}^{\text {Plan }}\left(\xi_{1} \leq x_{1}\right)\right) d x_{1} \\
& \leq \liminf _{N \rightarrow \infty}\left[T^{a}\left(1-\mathbb{P}_{N}^{\text {Plan }}\left(\xi_{1} \leq T\right)\right)+\int_{T}^{\infty} a x_{1}^{a-1}\left(1-\mathbb{P}_{N}^{\text {Plan }}\left(\xi_{1} \leq x_{1}\right)\right) d x_{1}\right] \\
& =\liminf _{N \rightarrow \infty} \mathbb{E}_{N}^{\text {Plan }}\left(\xi_{1}^{a} \chi_{\xi_{1}>T}\right) \\
& \leq C e^{-c T^{3 / 2}}<\infty .
\end{aligned}
$$

The proof of the finiteness of the second expected value in (3.13) is similar using (4.9).

\section{Tail estimates}

For the proof of Theorem 3.1, we need tail estimates for the (scaled) length $\xi_{k}$ of each row, which are uniform in $N$. In this section, we obtain these tail estimates in Proposition 4.3. These estimates follow from the tail estimates, Proposition 4.1 for the Poissonized Plancherel measure introduced in Section 1., together with the de-Poissonization Lemma 4.2.

Define

$$
\phi_{n}^{(k)}(t):=\mathbb{P}_{t}^{\text {Pois }}\left(\lambda_{k} \leq n\right)=\sum_{N=0}^{\infty} \frac{e^{-t^{2}} t^{2 N}}{N !} \mathbb{P}_{N}^{\text {Plan }}\left(\lambda_{k} \leq n\right) .
$$

(In [3, 田, the notation $\lambda=\sqrt{t}$ is used. But in this paper, to avoid the confusion with the notation $\lambda$ for a partition, we use $t$.) The following result is proved in Section 5 using the steepest-descent method for RHP. Note that $0 \leq \phi_{n}^{(k)}(t) \leq 1$. 
Proposition 4.1. Define $x$ by

$$
\frac{2 t}{n}=1-\frac{x}{2^{1 / 3} n^{2 / 3}}
$$

Let $k \in \mathbb{N}$. There are constants $C, c>0$ and $0<\delta_{0}<1$ such that for large $t$ and $n$, and for any fixed $0<\delta<\delta_{0}$, the following hold true : for $x \geq 0$,

$$
\begin{array}{cc}
0 \leq 1-\phi_{n}^{(k)}(t) \leq C e^{-c n}, & 0 \leq \frac{2 t}{n} \leq 1-\delta, \\
0 \leq 1-\phi_{n}^{(k)}(t) \leq C e^{-c x^{3 / 2}}, & 1-\delta<\frac{2 t}{n} \leq 1,
\end{array}
$$

and for $x<0$,

$$
\begin{array}{cl}
0 \leq \phi_{n}^{(k)}(t) \leq C e^{-c|x|^{3 / 2}}, & 1<\frac{2 t}{n}<1+\delta, \\
0 \leq \phi_{n}^{(k)}(t) \leq C e^{-c t}, & 1+\delta \leq \frac{2 t}{n} .
\end{array}
$$

We also need the following de-Poissonization lemma :

Lemma 4.2. There exists $C>0$ such that for all sufficiently large $N$,

$$
\mathbb{P}_{N}^{\text {Plan }}\left(\lambda_{k} \leq n\right) \leq C \phi_{n}^{(k)}\left((N-\sqrt{N})^{1 / 2}\right), \quad 1-\mathbb{P}_{N}^{\text {Plan }}\left(\lambda_{k} \leq n\right) \leq C\left(1-\phi_{n}^{(k)}\left((N+\sqrt{N})^{1 / 2}\right)\right)
$$

for all $n \in \mathbb{Z}$.

Proof. This is similar to Lemma 8.3 in [3] (again note that $\lambda$ in [3] satisfies $\lambda=\sqrt{t}$.) Indeed, the proof of Lemma 8.3 in [3] only requires the fact that $0 \leq q_{n, N+1} \leq q_{n, N} \leq 1$. In our case, $q_{n, N}=\mathbb{P}_{N}^{\text {Plan }}\left(\lambda_{k} \leq n\right)$, which is clearly between 0 and 1 . The monotonicity can be found in [24] Lemma 3.8.

Now Proposition 4.1 and Lemma 4.2 imply the following uniform tail estimates.

Proposition 4.3. Fix $k \in \mathbb{N}$ and $a \in \mathbb{N}_{0}$. For a given $T \geq 2$, there are constants $C, c>0$ and $N_{0}>0$ such that for $N \geq N_{0}$,

$$
\mathbb{E}_{N}^{\mathrm{Plan}}\left(\xi_{k}^{a} \chi_{\xi_{k}>T}\right) \leq C e^{-c T^{3 / 2}}+C e^{-c N^{1 / 2}}
$$

and

$$
\mathbb{E}_{N}^{\mathrm{Plan}}\left(\left|\xi_{k}\right|^{a} \chi_{\xi_{k}<-T}\right) \leq C e^{-c T^{3 / 2}}+C e^{-c N^{1 / 2}}
$$

Proof. (a) Bound (4.8) : Without any loss we can assume $a>0$. Note that since $0 \leq \lambda_{k} \leq N$,

$$
-2 N^{1 / 3} \leq \xi_{k} \leq \frac{N-2 \sqrt{N}}{N^{1 / 6}}<N^{5 / 6}
$$


If $T \geq N^{5 / 6}$, then the expected value in (4.8) is zero, and the bound is trivial. Thus we assume that $T<N^{5 / 6}$. Integrating by parts and using Lemma 4.2 ,

$$
\begin{aligned}
\mathbb{E}_{N}^{\text {Plan }}\left(\xi_{k}^{a} \chi_{\xi_{k}>T}\right) & =\int_{\left(T, N^{5 / 6}\right)} s^{a} d \mathbb{P}_{N}^{\text {Plan }}\left(\xi_{k} \leq s\right) \\
& =T^{a}\left(1-\mathbb{P}_{N}^{\text {Plan }}\left(\xi_{k} \leq T\right)\right)+\int_{\left(T, N^{5 / 6}\right)} a s^{a-1}\left(1-\mathbb{P}_{N}^{\text {Plan }}\left(\xi_{k} \leq s\right)\right) d s \\
& \leq C T^{a}\left(1-\phi_{n(T)}^{(1)}\left((N+\sqrt{N})^{1 / 2}\right)\right)+C \int_{\left(T, N^{5 / 6}\right)} a s^{a-1}\left(1-\phi_{n(s)}^{(1)}\left((N+\sqrt{N})^{1 / 2}\right)\right) d s
\end{aligned}
$$

for large $N$, where $n(s)=2 \sqrt{N}+s N^{1 / 6}$. Note that since $T \geq 2, \frac{2(N+\sqrt{N})^{1 / 2}}{n(T)} \leq 1$. We distinguish two cases :

$$
\begin{aligned}
& \text { (i) } 0 \leq \frac{2(N+\sqrt{N})^{1 / 2}}{n(T)}<1-\delta \\
& \text { (ii) } 1-\delta \leq \frac{2(N+\sqrt{N})^{1 / 2}}{n(T)} \leq 1
\end{aligned}
$$

where $0<\delta<1$ is a fixed constant satisfying $\delta<\delta_{0}$, where $\delta_{0}$ appears in Proposition 4.1.

Case (i) : For all $s \geq T, 0 \leq \frac{2(N+\sqrt{N})^{1 / 2}}{n(s)} \leq \frac{2(N+\sqrt{N})^{1 / 2}}{n(T)}<1-\delta$. Note that for $T \leq s$,

$$
n(s) \geq n(T) \geq \frac{2(N+\sqrt{N})^{1 / 2}}{1-\delta} \geq \frac{2 \sqrt{N}}{1-\delta}
$$

Hence from the estimate (4.3), we have

$$
1-\phi_{n(s)}^{(1)}\left((N+\sqrt{N})^{1 / 2}\right) \leq C e^{-c n(s)} \leq C e^{-c N^{1 / 2}}
$$

for $T \leq s<N^{5 / 6}$ with a new constant $c$. Therefore, from (4.11), we obtain

$$
\mathbb{E}_{N}^{\mathrm{Plan}}\left(\xi_{k}^{a} \chi_{\xi_{k}>T}\right) \leq C e^{-c N^{1 / 2}}
$$

Case (ii) : There is $s_{0}>T$ such that $\frac{2(N+\sqrt{N})^{1 / 2}}{n\left(s_{0}\right)}=1-\delta$. We write 4.11) as

$$
\begin{aligned}
\mathbb{E}_{N}^{\text {Plan }}\left(\xi_{k}^{a} \chi_{\xi_{k}>T}\right) \leq & C T^{a}\left(1-\phi_{n(T)}^{(1)}\left((N+\sqrt{N})^{1 / 2}\right)\right)+C \int_{\left(T, s_{0}\right)} a s^{a-1}\left(1-\phi_{n(s)}^{(1)}\left((N+\sqrt{N})^{1 / 2}\right)\right) d s \\
& +C \int_{\left[s_{0}, N^{5 / 6}\right)} a s^{a-1}\left(1-\phi_{n(s)}^{(1)}\left((N+\sqrt{N})^{1 / 2}\right)\right) d s \quad\left(\text { if } s_{0} \geq N^{5 / 6}, \text { the third integral is zero }\right) \\
\leq & C T^{a} e^{-c x(T)^{3 / 2}}+C \int_{\left(T, s_{0}\right)} a s^{a-1} e^{-c x(s)^{3 / 2}} d s+C \int_{\left[s_{0}, N^{5 / 6}\right)} a s^{a-1} e^{-c n(s)} d s
\end{aligned}
$$

using (4.3), (4.4), where $x(s)$ is defined by the formula (4.2) with $t=(N+\sqrt{N})^{1 / 2}$ and $n=2 \sqrt{N}+s N^{1 / 6}$. As in Case (i), for $s \geq s_{0}$,

$$
n(s) \geq n\left(s_{0}\right)=\frac{2(N+\sqrt{N})^{1 / 2}}{1-\delta} \geq \frac{2 \sqrt{N}}{1-\delta}
$$


and hence, the last integral is less than $C e^{-c N^{1 / 2}}$. For the other terms, since

$$
2 \sqrt{N}-2(N+\sqrt{N})^{1 / 2}=\frac{-4 \sqrt{N}}{2 \sqrt{N}+2(N+\sqrt{N})^{1 / 2}} \geq-1 \geq-N^{1 / 6},
$$

we have for $T \leq s<s_{0}$,

$$
x(s)=\frac{n-2 t}{(n / 2)^{1 / 3}}=\frac{2 \sqrt{N}+s N^{1 / 6}-2(N+\sqrt{N})^{1 / 2}}{\left(\sqrt{N}+\frac{s}{2} N^{1 / 6}\right)^{1 / 3}} \geq \frac{s-1}{\left(1+\frac{s}{2 N^{1 / 3}}\right)^{1 / 3}} \geq \frac{\frac{1}{2}}{\left(1+\frac{s}{2 N^{1 / 3}}\right)^{1 / 3}}
$$

as $s \geq T \geq 2$. Noting that $s_{0}=\frac{2(N+\sqrt{N})^{1 / 2}-2(1-\delta) \sqrt{N}}{(1-\delta) N^{1 / 6}} \leq c_{0} N^{1 / 3}$ for some constant $c_{0}$, we have

$$
x(s) \geq \frac{\frac{1}{2}}{\left(1+\frac{s}{2 N^{1 / 3}}\right)^{1 / 3}} \geq c s
$$

for $s \leq s_{0}$ with some constant $c>0$. Hence

$$
\begin{aligned}
\mathbb{E}_{N}^{\mathrm{Plan}}\left(\xi_{k}^{a} \chi_{\xi_{k}>T}\right) & \leq C T^{a} e^{-c T^{3 / 2}}+C \int_{T}^{\infty} s^{a-1} e^{-c s^{3 / 2}} d s+C e^{-c N^{1 / 2}} \\
& \leq C e^{-c T^{3 / 2}}+C e^{-c N^{1 / 2}}
\end{aligned}
$$

(b) Bound (4.9) : Recalling (4.10), if $T \geq 2 N^{1 / 3}$, the expected value in (4.9) is zero and the bound is trivial. Thus we assume that $T \leq 2 N^{1 / 3}$. Integrating by parts and using Lemma 1.2, we have for some constants $C, c>0$,

$$
\begin{aligned}
\mathbb{E}_{N}^{\mathrm{Plan}}\left(\left|\xi_{k}^{a}\right| \chi_{\xi_{k}<-T}\right) & =\int_{\left[-2 N^{1 / 3},-T\right)}(-s)^{a} d \mathbb{P}_{N}^{\mathrm{Plan}}\left(\xi_{k} \leq s\right) \\
& =|-T|^{a} \mathbb{P}_{N}^{\mathrm{Plan}}\left(\xi_{k}<-T\right)+\int_{\left[-2 N^{1 / 3},-T\right)} a(-s)^{a-1} \mathbb{P}_{N}^{\mathrm{Plan}}\left(\xi_{k} \leq s\right) d s \\
& \leq C T^{a} \phi_{n(-T)}^{(k)}\left((N-\sqrt{N})^{1 / 2}+C \int_{\left[-2 N^{1 / 3},-T\right)} a(-s)^{a-1} \phi_{n(s)}^{(k)}\left((N-\sqrt{N})^{1 / 2} d s\right.\right.
\end{aligned}
$$

for large $N$, where $n(s)=2 \sqrt{N}+s N^{1 / 6}$ as before. Given $T$, we take $N_{0}>\frac{1+\sqrt{1+T^{3}}}{2 T}$ so that for $N \geq N_{0}$, $\frac{2(N-\sqrt{N})^{1 / 2}}{n(-T)}>1$. We distinguish two cases :

$$
\begin{aligned}
& \text { (i) } \quad 1+\delta \leq \frac{2(N-\sqrt{N})^{1 / 2}}{n(-T)} \\
& \text { (ii) } 1<\frac{2(N-\sqrt{N})^{1 / 2}}{n(-T)}<1+\delta
\end{aligned}
$$

where $0<\delta<1$ is a fixed constant as above.

Case (i) : For all $-2 N^{1 / 3}-2 N^{1 / 3}<s<-T, \frac{2(N-\sqrt{N})^{1 / 2}}{n(s)} \geq \frac{2(N-\sqrt{N})^{1 / 2}}{n(-T)} \geq 1+\delta$. From the estimate (4.6), using $T \leq 2 N^{1 / 3}$,

$$
\mathbb{E}_{N}^{\mathrm{Plan}}\left(\left|\xi_{k}^{a}\right| \chi_{\xi_{k}<-T}\right) \leq C e^{-c N^{1 / 2}}
$$


Case (ii) : There is $s_{0}>T$ such that $\frac{2(N-\sqrt{N})^{1 / 2}}{n\left(-s_{0}\right)}=1+\delta$. We write 4.23$)$ as

$$
\begin{aligned}
\mathbb{E}_{N}^{\mathrm{Plan}}\left(\left|\xi_{k}^{a}\right| \chi_{\xi_{k}<-T} \leq \leq\right. & C T^{a} \phi_{n(-T)}^{(k)}\left((N-\sqrt{N})^{1 / 2}\right)+C \int_{\left(-s_{0},-T\right)} a(-s)^{a-1} \phi_{n(-T)}^{(k)}\left((N-\sqrt{N})^{1 / 2}\right) d s \\
& +C \int_{\left[-2 N^{1 / 3},-s_{0}\right]} a(-s)^{a-1} \phi_{n(-T)}^{(k)}\left((N-\sqrt{N})^{1 / 2}\right) d s \\
\leq & C T^{a} e^{-c|y(-T)|^{3 / 2}}+C \int_{\left(-s_{0},-T\right)} a(-s)^{a-1} e^{-c|y(s)|^{3 / 2}} d s \\
& +C \int_{\left[-2 N^{1 / 3},-s_{0}\right]} a(-s)^{a-1} e^{-c(N-\sqrt{N})^{1 / 2}} d s
\end{aligned}
$$

using (4.5), (4.6), where $y(s)$ is defined by $x$ in the formula (4.2) with $t=(N-\sqrt{N})^{1 / 2}$ and $n=$ $2 \sqrt{N}+s N^{1 / 6}$. The last integral is less than $C e^{-c N^{1 / 2}}$ with new constants $C, c$. For the other terms, note that since $2 \sqrt{N}-2(N-\sqrt{N})^{1 / 2} \leq 2 \leq N^{1 / 6}$ and $s \leq-T \leq-2$, we have for $-2 N^{1 / 3} \leq s \leq-T$,

$$
y(s)=\frac{n-2 t}{(n / 2)^{1 / 3}}=\frac{s+\left[2 \sqrt{N}-2(N-\sqrt{N})^{1 / 2}\right] N^{-1 / 6}}{\left(1+s /\left(2 N^{1 / 3}\right)\right)^{1 / 3}} . \leq s+1 \leq \frac{s}{2} .
$$

Thus, we obtain

$$
\begin{aligned}
\mathbb{E}_{N}^{\mathrm{Plan}}\left(\left|\xi_{k}^{a}\right| \chi_{\xi_{k}<-T}\right) & \leq C T^{a} e^{-c T^{3 / 2}}+C \int_{\left(-s_{0},-T\right)} a|s|^{a-1} e^{-c|s|^{3 / 2}} d s+C e^{-c N^{1 / 2}} \\
& \leq C e^{-c T^{3 / 2}}+C e^{-c N^{1 / 2}}
\end{aligned}
$$

Remark 4.4. The results (4.3) - (4.6) for $k=1$ were given in [3]. Indeed, in [3] stronger bounds than (4.5), (4.6) were obtained (Lemma 7.1 (iv), (v) in 司) :

$$
\begin{array}{cl}
0 \leq \phi_{n}^{(1)}(t) \leq C e^{-c|x|^{3}}, & 1<\frac{2 t}{n}<1+\delta, \\
0 \leq \phi_{n}^{(1)}(t) \leq C e^{-c t^{2}}, & 1+\delta \leq \frac{2 t}{n}
\end{array}
$$

(note $\lambda=\sqrt{t}$ in [3].) From this, as in the Proof of Proposition 4.9. we have

$$
\mathbb{E}_{N}^{\mathrm{Plan}}\left(\left|\xi_{1}^{a}\right| \chi_{\xi_{1}<-T}\right) \leq C e^{-c T^{3}}+C e^{-c N} .
$$

In this paper, we only obtain the above weaker bounds (4.5) and (4.6), but they are enough for our purpose in proving the convergence of moments. However, we believe that the same bound (4.32) holds true for general $k$. In the next section, we indicate why we only obtain these weaker bounds (see the Remark before Lemma 5.9 below). 


\section{Riemann-Hilbert problem}

In this section, we will prove Proposition 4.1.

For (4.3) and (4.4), note that $\phi_{n}^{(k)}(t) \geq \phi_{n}^{(1)}(t)$ for all $k \geq 1$ as $\lambda_{1} \geq \lambda_{2} \geq \cdots$. But for $k=1$ the estimates (4.3), (4.4) were proved in [3] (Lemma 7.1 (i), (ii)), and so we have the same bounds for all $k \geq 1$. On the other hand, since $\mathbb{P}_{N}^{\text {Plan }}(\lambda \leq n) \leq 1$, we always have $0 \leq \phi_{n}^{(k)}(t) \leq 1$.

The rest of this section is devoted to proving (4.5) and 4.6). We start from the formulae (see [8, 24, 28, 31]) that $\left(\right.$ recall $\left.\phi_{n}^{(k)}(t)=\mathbb{P}_{t}^{\text {Pois }}\left(\lambda_{k} \leq n\right)\right)$

$$
\begin{aligned}
\phi_{n}^{(1)}(t) & =\operatorname{det}\left(1-\mathrm{S}_{\mathrm{n}}\right), \\
\phi_{n+k}^{(k+1)}(t) & =\phi_{n+k-1}^{(k)}(t)+\left.\left(-\frac{d}{d r}\right)^{k}\right|_{r=1} \operatorname{det}\left(1-r \mathrm{~S}_{\mathrm{n}}\right), \quad k \geq 1,
\end{aligned}
$$

with

$$
\varphi(z)=e^{t\left(z-z^{-1}\right)} .
$$

This follows from, for example, Theorem 3.1 of [31] with $p_{+}=p_{-}=(t, 0,0)$ (see also [30]) which states that for any finite subset $A$ of $\mathbb{Z}$,

$$
\mathbb{P}_{t}^{\text {Pois }}\left(A \subset\left\{\lambda_{j}-j\right\}\right)=\operatorname{det}(S(i, j))_{i, j \in A}, \quad S(i, j)=\sum_{k=1}^{\infty}\left(\varphi^{-1}\right)_{i+k} \varphi_{-j-k},
$$

with $\varphi$ given by (5.3), where $\mathbb{P}_{t}^{\text {Pois }}$ denotes the Plancherel measure for $S_{N}$ with $N$ being the Poisson variable with mean $t^{2}$ (Poissonized Plancherel measure). Recall from 4.1$)$ that $\phi_{n}^{(k)}(t)=\mathbb{P}_{t}^{\text {Pois }}\left(\lambda_{k} \leq n\right)$. In [3], the authors obtained the estimates (stronger than) (4.5) and (4.6) in the case $k=1$ (see the Remark at the end of Section $\mathbb{1}$. Hence we need to prove that for any fixed $k \in \mathbb{N}$,

$$
\begin{aligned}
\left|\frac{d^{k}}{d r^{k}}\right|_{r=1} \operatorname{det}\left(1-r \mathrm{~S}_{\mathrm{n}}\right) \mid \leq C e^{-c|x|^{3 / 2},} & 1<\frac{2 t}{n}<1+\delta, \\
\left|\frac{d^{k}}{d r^{k}}\right|_{r=1} \operatorname{det}\left(1-r \mathrm{~S}_{\mathrm{n}}\right) \mid \leq C e^{-c t}, & 1+\delta \leq \frac{2 t}{n} .
\end{aligned}
$$

By Cauchy's theorem, we write for $0<\epsilon<1$,

$$
\left.\frac{d^{k}}{d r^{k}}\right|_{r=1} \operatorname{det}\left(1-r \mathrm{~S}_{\mathrm{n}}\right)=\frac{k !}{2 \pi i} \int_{|s-1|=\epsilon} \frac{\operatorname{det}\left(1-s \mathrm{~S}_{\mathrm{n}}\right)}{(s-1)^{k+1}} d s .
$$

By Remark 3 in Section 2, for $\varphi(z)$ as in (5.3), $\mathrm{S}_{\mathrm{n}}$ is positive and $\left\|\mathrm{S}_{\mathrm{n}}\right\| \leq 1$. Hence the eigenvalues $a_{j}$ of (the trace class operator) $\mathrm{S}_{\mathrm{n}}$ satisfies $0 \leq a_{j} \leq 1$ (actually one can show that $0 \leq a_{j}<1$ ). For $|s-1|=\epsilon$,

$$
\left|\operatorname{det}\left(1-s \mathrm{~S}_{\mathrm{n}}\right)\right|=\prod_{j}\left|1-s a_{j}\right| \leq \prod_{j}\left(1-(1-\epsilon) a_{j}\right)=\operatorname{det}\left(1-(1-\epsilon) \mathrm{S}_{\mathrm{n}}\right) .
$$

Therefore we have

$$
\left|\frac{d^{k}}{d r^{k}}\right|_{r=1} \operatorname{det}\left(1-r \mathrm{~S}_{\mathrm{n}}\right) \mid \leq \frac{k !}{\epsilon^{k}} \operatorname{det}\left(1-(1-\epsilon) \mathrm{S}_{\mathrm{n}}\right) .
$$


Thus it is sufficient to prove that for fixed $0<r<1$,

$$
\begin{aligned}
\operatorname{det}\left(1-r \mathrm{~S}_{\mathrm{n}}\right) \leq C e^{-c|x|^{3 / 2},} & 1<\frac{2 t}{n}<1+\delta, \\
\operatorname{det}\left(1-r \mathrm{~S}_{\mathrm{n}}\right) \leq C e^{-c t}, & 1+\delta \leq \frac{2 t}{n},
\end{aligned}
$$

or by Theorem 2.1, we need to prove that for any fixed $0<s<1$, (note that $\varphi$ given by (5.3) has no winding)

$$
\begin{array}{cl}
(1+s)^{-n} \operatorname{det}\left(1-s \mathrm{~K}_{\mathrm{n}}\right) \leq C e^{-c|x|^{3 / 2},} & 1<\frac{2 t}{n}<1+\delta, \\
(1+s)^{-n} \operatorname{det}\left(1-s \mathrm{~K}_{\mathrm{n}}\right) \leq C e^{-c t}, & 1+\delta \leq \frac{2 t}{n},
\end{array}
$$

where $x$ is defined in (4.2)

$$
\frac{2 t}{n}=1-\frac{x}{2^{1 / 3} n^{2 / 3}}
$$

Since $\mathrm{K}_{\mathrm{n}}$ is an integrable operator, there is a naturally associated Riemann-Hilbert problem (see [21, 12). Let $m(z ; k)$ be the $2 \times 2$ matrix function which solves the following Riemann-Hilbert problem (RHP) : with contour $\Sigma=\{|z|=1\}$, oriented counterclockwise,

$$
\left\{\begin{array}{l}
m(z ; k) \quad \text { is analytic in } z \in \mathbb{C} \backslash \Sigma, \\
m_{+}(z ; k)=m_{-}(z ; k)\left(\begin{array}{cc}
1-s^{2} & -s z^{-k} e^{-t\left(z-z^{-1}\right)} \\
s z^{k} e^{t\left(z-z^{-1}\right)} & 1 .
\end{array}\right) \\
m(z ; k) \rightarrow I \quad \text { as } z \rightarrow \infty .
\end{array} \quad \text { for } z \in \Sigma,\right.
$$

Here and also in the following, the notation $f_{+}(z)$ (resp., $f_{-}(z)$ ) denotes the limiting value of $\lim _{z^{\prime} \rightarrow z} f\left(z^{\prime}\right)$ from the left (resp., right) of the contour in the direction of the orientation. In the above case, $m_{+}(z ; k ; s)$ (resp., $\left.m_{-}(z ; k ; s)\right)$ means $\lim _{z^{\prime} \rightarrow z} m\left(z^{\prime} ; k ; s\right)$ with $\left|z^{\prime}\right|<1$ (resp., $\left|z^{\prime}\right|>1$.) In (52) of [四, it is shown that

$$
(1+s)^{-n} \operatorname{det}\left(1-s \mathrm{~K}_{\mathrm{n}}\right)=\prod_{k=n}^{\infty} m_{11}(0 ; k+1)
$$

where $m_{11}(0 ; k)$ is the (11)-entry of $m(z ; k)$ evaluated at $z=0$. Therefore, in order to prove (5.12) and (5.13), we need asymptotic results for $m_{11}(0 ; k)$ as $k, t \rightarrow \infty$. In the special case when $s=1$, this RHP is algebraically equivalent to the RHP for the orthogonal polynomials on the unit circle with respect to the measure $e^{t\left(z+z^{-1}\right)} d z /(2 \pi i z)$, whose asymptotics as $k, t \rightarrow \infty$ was investigated in [3]. The RHP (5.15) was introduced in 迎.

There is a critical difference in the asymptotic analysis depending whether $s=1$ or $0<s<1$. In the former case, the jump matrix in (5.15) has a upper/lower factorization, but not a lower/upper factorization, while in the later case, the jump matrix has both factorizations. This difference makes the later case much easier to analyze asymptotically. In the former case, we need a WKB type analysis which involves the construction of a parametrix in terms of the equilibrium measure of a certain variational 
problem, and in the case where $\frac{2 t}{n}>1$, the main asymptotic contribution to the RHP comes from the part of the circle near $z=-1$. But in the later case, due to the existence of both factorization of the jump matrix, the RHP localizes in the limit just to two points on the circle. We refer the reader to [16] for an example of the second type, and to [17, 15, 14] for examples of the first type.

Remark 5.1. The different analysis for $s=1$ and $0<s<1$ gives us different estimates. Indeed, when $s=1$, instead of (5.18) below, we have (see (6.42) of 河)

$$
\log m_{11}(0 ; k) \leq k\left(-\frac{2 t}{k}+\log \frac{2 t}{k}+1\right)
$$

which imply (4.30), (4.31). Thus, in order to obtain the better estimates (4.30), (4.31), (4.32) for general row $\xi_{k}$ (see Remark at the end of Section 4 ), we need to analysis the RHP (5.15) as $s \rightarrow 1$ instead of fixed $s<1$.

Fix $0<s<1$. We will prove the following estimate.

Lemma 5.2. There are positive constants $M_{0}$ and $c$ such that when $\frac{2 t}{k} \geq 1+\frac{M_{0}}{2^{1 / 3} k^{2 / 3}}$, we have

$$
\log m_{11}(0 ; k) \leq-c \sqrt{1-\left(\frac{k}{2 t}\right)^{2}}
$$

for large $t$.

Assuming this result, we will prove (5.12) and (5.13), which completes the proof of Proposition 4.1. Proof of Proposition 4.1. We need to prove (5.12) and (5.13).

(a) Estimate (5.12) : It is enough to prove (5.12) for $-2^{1 / 3} n^{2 / 3} \delta<x \leq-M$ where $M:=$ $M_{0}(1+2 \delta)$ with $M_{0}$ in Lemma 5.2. As noted above, since $\mathrm{S}_{\mathrm{n}}$ is positive and $\left\|\mathrm{S}_{\mathrm{n}}\right\| \leq 1$, its eigenvalues $a_{j}$ satisfies $0 \leq a_{j} \leq 1$. Hence for $0<s<1$, by (5.16) and Theorem 2.1,

$$
0 \leq \prod_{k=n}^{\infty} m_{11}(0 ; k+1)=\operatorname{det}\left(1-s^{2} \mathrm{~S}_{\mathrm{n}}\right)=\prod_{j}\left(1-s^{2} a_{j}\right) \leq 1,
$$

for any $n$. Therefore in order to prove (5.12), it is enough to show that

$$
\sum_{(*)} \log m_{11}(0 ; k+1) \leq-c|x|^{3 / 2}+C
$$

for some constants $c, C>0$ where the summation is over the set (note $x+M \leq 0$ in (5.20))

$$
(*): \quad n \leq k \leq n-\frac{x+M_{1}}{2^{1 / 3}} n^{1 / 3},
$$

with $M_{1}:=M_{0}(1+\delta)$. We will show that for $k$ in $(*)$,

$$
1+\frac{M_{0}}{2^{1 / 3} k^{2 / 3}} \leq \frac{2 t}{k}
$$


Since $n \leq k, 5.22$ follows from

$$
\frac{2 t}{k} \geq 1+\frac{M_{0}}{2^{1 / 3} n^{2 / 3}} .
$$

In order to show (5.23), since

$$
\frac{2 t}{k}=\frac{2 t}{n} \cdot \frac{n}{k} \geq\left(1-\frac{x}{2^{1 / 3} n^{2 / 3}}\right)\left(1-\frac{x+M_{1}}{2^{1 / 3} n^{2 / 3}}\right)^{-1},
$$

it is enough to checking that

$$
\left(1-\frac{x}{2^{1 / 3} n^{2 / 3}}\right) \geq\left(1-\frac{x+M_{1}}{2^{1 / 3} n^{2 / 3}}\right)\left(1+\frac{M_{0}}{2^{1 / 3} n^{2 / 3}}\right),
$$

which is equivalent to check that

$$
M_{1} \geq\left(1-\frac{x+M_{1}}{2^{1 / 3} n^{2 / 3}}\right) M_{0} .
$$

But since $-x \leq 2^{1 / 3} \delta n^{2 / 3}$,

$$
\left(1-\frac{x+M_{1}}{2^{1 / 3} n^{2 / 3}}\right) M_{0} \leq\left(1+\delta-\frac{M_{1}}{2^{1 / 3} n^{2 / 3}}\right) \frac{M_{1}}{1+\delta} \leq M_{1},
$$

and hence $(5.22)$ is proved.

Now using (5.18), the sum in (5.20) satisfies

$$
\begin{aligned}
\sum_{(*)} \log m_{11}(0 ; k+1) & \leq \sum_{(*)}-c \sqrt{1-\left(\frac{k}{2 t}\right)^{2}} \\
& \leq \sum_{(*)}-c \sqrt{1-\frac{k}{2 t}} \\
& \leq-c \int_{n}^{n-\frac{x+M_{1}}{2^{1 / 3}} n^{1 / 3}} \sqrt{1-\frac{s}{2 t}} d s \\
& =c\left(\frac{4 t}{3}\right)\left[\left(1-\frac{n-\frac{x+M_{1}}{2^{1 / 3}} n^{1 / 3}}{2 t}\right)^{3 / 2}-\left(1-\frac{n}{2 t}\right)^{3 / 2}\right] \\
& =\frac{2}{3} c\left(\frac{2 t}{n}\right)^{-1 / 2}\left[\left(n^{2 / 3}\left(\frac{2 t}{n}-1\right)+\frac{x+M_{1}}{2^{1 / 3}}\right)^{3 / 2}-\left(n^{2 / 3}\left(\frac{2 t}{n}-1\right)\right)^{3 / 2}\right] \\
& =\frac{2}{3} c\left(\frac{2 t}{n}\right)^{-1 / 2}\left[\left(\frac{M_{1}}{2^{1 / 3}}\right)^{3 / 2}-\left(\frac{-x}{2^{1 / 3}}\right)^{3 / 2}\right]
\end{aligned}
$$

where the second inequality is due to the monotonicity of the function $f(y)=\sqrt{1-\frac{y}{2 t}}$. Since $1<\frac{2 t}{n}<$ $1+\delta$, we obtain (5.12).

(b) Estimate (5.13) : By a similar argument as in (a), it is enough to show that

$$
\sum_{(* *)} \log m_{11}(0 ; k+1) \leq-c t+C,
$$

for some constants $c, C>0$ where $(* *)$ is the set

$$
(* *): \quad \frac{2 t}{1+\delta} \leq k \leq \frac{2 t}{1+\delta / 2} .
$$


For $k$ in $(* *)$, we have

$$
\frac{k}{2 t} \leq \frac{1}{1+\delta / 2}<1
$$

Now using (5.18), the sum in (5.29) satisfies

$$
\begin{aligned}
\sum_{(* *)} \log m_{11}(0 ; k+1) & \leq \sum_{(* *)}-c \sqrt{1-\left(\frac{k}{2 t}\right)^{2}} \\
& \leq-c \sqrt{1-\left(\frac{1}{1+\delta / 2}\right)^{2}}\left(\frac{2 t}{1+\delta / 2}-\frac{2 t}{1+\delta}+1\right) \\
& \leq-c t+C .
\end{aligned}
$$

\section{RHP Asymptotics and Proof of Lemma 5.2}

In the rest of this section, we prove Lemma 5.2 by asymptotic analysis of the RHP (5.15).

Set

$$
\eta:=\frac{k}{2 t}
$$

Under the condition of Lemma 5.2, we have $\eta<1$. We denote by $v(z)$ the jump matrix in the second condition of the RHP (5.15). Note that the (21)-entry of $v$ is $s e^{2 t f(z ; \eta)}$ where

$$
f(z ; \eta):=\frac{1}{2}\left(z-z^{-1}\right)+\eta \log z,
$$

where $\log z \in \mathbb{R}$ for $z>0$. The critical points of this function are $\xi:=e^{i \theta_{c}}$ and $\xi^{-1}=\bar{\xi}$ where

$$
\xi=-\eta+i \sqrt{1-\eta^{2}}
$$

Note that $-\pi / 2<\theta_{c}<\pi$. For $z=\rho e^{i \theta}$, consider

$$
F_{\theta}(\rho):=\operatorname{Ref}(z)=\frac{1}{2}\left(\rho-\rho^{-1}\right) \cos \theta+\eta \log \rho .
$$

Its derivative at $\rho=1$ is

$$
\frac{d}{d \rho} F_{\theta}(1)=\cos \theta+\eta
$$

which is positive for $|\theta|<\theta_{c}$, and is negative for $\theta_{c}<|\theta| \leq \pi$. Indeed it is easy to check that :

(i). When $|\theta| \leq \frac{\pi}{2}, F_{\theta}(\rho)<0$ for $0<\rho<1$, and $F_{\theta}(\rho)>0$ for $\rho>1$.

(ii). When $\frac{\pi}{2}<|\theta|<\theta_{c}, F_{\theta}(\rho)>0$ for $0<\rho<\rho_{0}, F_{\theta}(\rho)<0$ for $\rho_{0}<\rho<1, F_{\theta}(\rho)>0$ for $1<\rho<\rho_{o}^{-1}$, and $F_{\theta}(\rho)<0$ for $\rho>\rho_{0}^{-1}$. Here $\rho_{0}$ is a number satisfying $0<\rho_{0}<\rho_{\theta}$, where

$$
\rho_{\theta}:=\frac{\eta-\sqrt{\eta^{2}-\cos ^{2} \theta}}{-\cos \theta}<1
$$

and $\frac{d}{d \theta} F_{\theta}\left(\rho_{\theta}\right)=\frac{d}{d \theta} F_{\theta}\left(\rho_{\theta}^{-1}\right)=0$. 

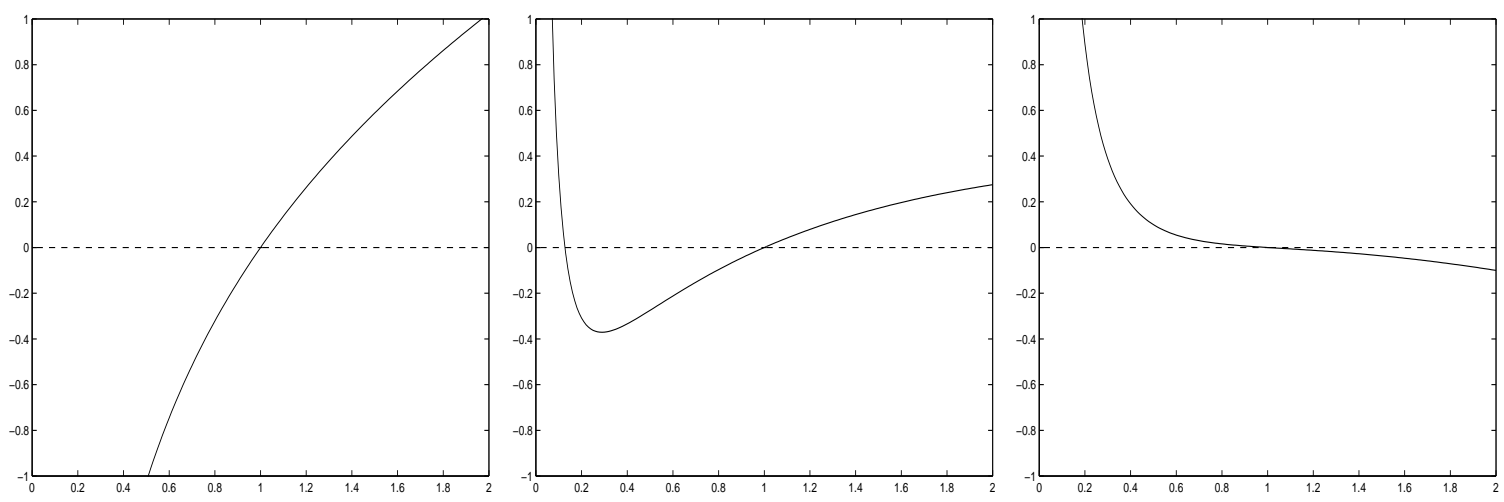

Figure 1: Graph of $F_{\theta}(\rho)$ for $\theta=\frac{\pi}{6}$ (case (i)), $\frac{5 \pi}{6}$ (case (ii)) and $\pi$ (case (iii)) when $\eta=15 / 16$

(iii). When $\theta_{c}<|\theta| \leq \pi, F_{\theta}(\rho)>0$ for $0<\rho<1$, and $F_{\theta}(\rho)<0$ for $\rho>1$.

(iv). The curve $\left\{\rho e^{i \theta}: F_{\theta}(\rho)=0\right\}$ crosses the circle at 90 degree.

Typical graphs of $F_{\theta}(\rho)$ is given in Figure 1 for the value $\eta=15 / 16$ for $\theta$ in the three different cases (i)-(iii). Figure 2 is a signature table for $\operatorname{Re}(f(z))$ when $\eta=15 / 16$. The solid curve is $\operatorname{Re}(f(z))=0$, and the dotted rays represent the lines $\cos \theta=-\eta$. The \pm signs denote the signature of $\operatorname{Re}(f(z))$ in each of the four components. The curve $\operatorname{Re}(f(z))=0$ and the lines $\cos \theta=-\eta$ meet on the unit circle at the points $\xi$ and $\xi^{-1}$.

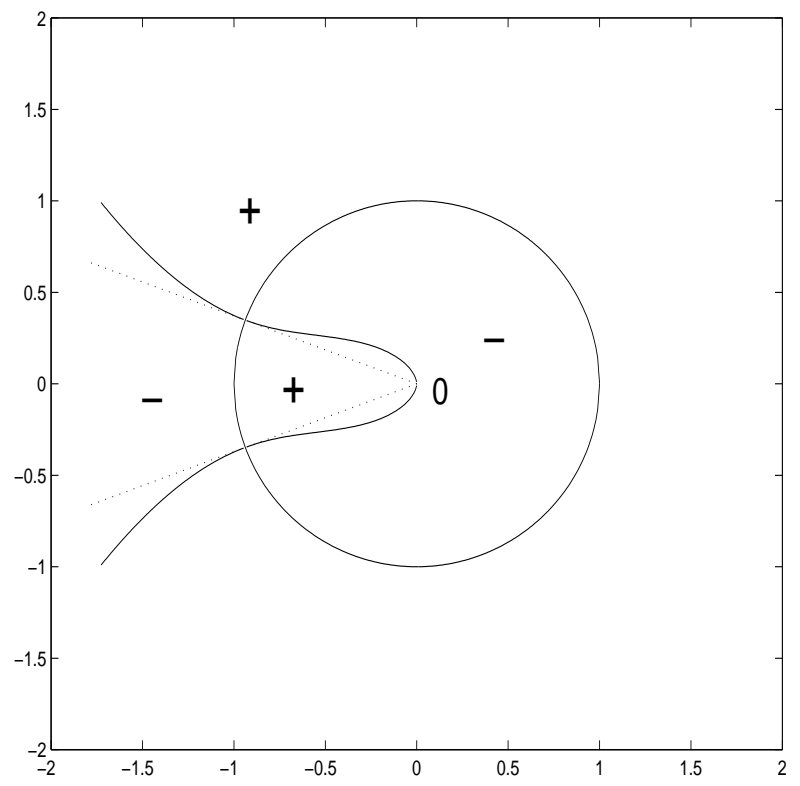

Figure 2: Curve $\operatorname{Re}(f(z))=0$ when $\eta=15 / 16$ 


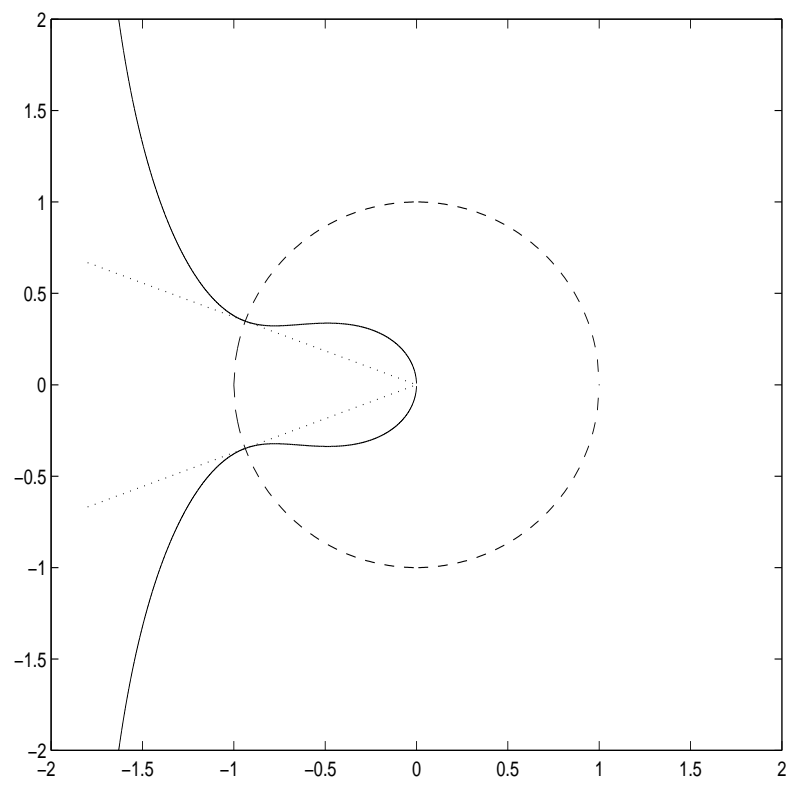

Figure 3: Solid curve represents the set $\left\{\rho_{\theta} e^{i \theta}: \frac{\pi}{2}<\theta \leq \theta_{c}\right\}$ when $\eta=15 / 16$

Let $\Sigma=\Sigma_{1} \cup \Sigma_{2}$, where $\Sigma_{1}=\left\{e^{i \theta}:|\theta|<\theta_{c}\right\}$, and $\Sigma_{2}=\Sigma \backslash \Sigma_{1}$. Define the function

$$
\delta(z):=\left(\frac{z-\xi}{z-\xi^{-1}}\right)^{-\frac{1}{2 \pi i} \log \left(1-s^{2}\right)},
$$

which is analytic in $\mathbb{C} \backslash \Sigma_{2}$ : we choose the branch so that $\delta(z) \rightarrow 1$ as $z \rightarrow \infty$ along the positive real axis. Then it solves the scalar Riemann-Hilbert problem

$$
\begin{aligned}
& \delta_{+}(z)=\delta_{-}(z)\left(1-s^{2}\right), \quad z \in \Sigma_{2}, \\
& \delta(z) \rightarrow 1, \quad \text { as } z \rightarrow \infty,
\end{aligned}
$$

where $\delta_{ \pm}$has the same meaning as in the RHP (5.15). Note that

$$
\delta(0)=\left(1-s^{2}\right)^{1-\frac{\theta_{c}}{\pi}} .
$$

Now set

$$
m^{(2)}(z):=m(z) \delta^{-\sigma_{3}}, \quad \sigma_{3}=\left(\begin{array}{cc}
1 & 0 \\
0 & -1
\end{array}\right) .
$$

Then (i) $m^{(2)}(z)$ is analytic in $\mathbb{C} \backslash \Sigma$, (ii) $m^{(2)}(z) \rightarrow I$ as $z \rightarrow \infty$, and (iii) $m_{+}^{(2)}(z)=m_{-}^{(2)} v^{(2)}(z)$ for $z \in \Sigma$, where

$$
v^{(2)}(z)=\left\{\begin{array}{lc}
\left(\begin{array}{cc}
1-s^{2} & -s e^{-2 t f(z)} \delta^{2}(z) \\
s e^{2 t f(z)} \delta^{-2}(z) & 1
\end{array}\right), & z \in \Sigma_{1}, \\
\left(\begin{array}{cc}
1 & -\frac{s}{1-s^{2}} e^{-2 t f(z)} \delta_{+}^{2}(z) \\
\frac{s}{1-s^{2}} e^{2 t f(z)} \delta_{-}^{-2} & 1-s^{2}
\end{array}\right), & z \in \Sigma_{2} .
\end{array}\right.
$$


Also we have

$$
m_{11}(0)=m_{11}^{(2)}(0)\left(1-s^{2}\right)^{1-\frac{\theta_{c}}{\pi}}
$$

Note that the jump matrix has the factorizations

$$
v^{(2)}(z)= \begin{cases}\left(\begin{array}{cc}
1 & -s e^{-2 t f(z)} \delta^{2}(z) \\
0 & 1
\end{array}\right)\left(\begin{array}{cc}
1 & 0 \\
s e^{2 t f(z)} \delta^{-2}(z) & 1
\end{array}\right), & z \in \Sigma_{1}, \\
\left(\begin{array}{cc}
1 & 0 \\
\frac{s}{1-s^{2}} e^{2 t f(z)} \delta_{-}^{-2} & 1-s^{2}
\end{array}\right)\left(\begin{array}{cc}
1 & -\frac{s}{1-s^{2}} e^{-2 t f(z)} \delta_{+}^{2}(z) \\
0 & 1
\end{array}\right), & z \in \Sigma_{2} .\end{cases}
$$

From (i)-(iii) in (5.38), we can take an oriented closed curve $\Sigma_{-}^{(3)}$ surrounding 0 and -1 , and passing through $\xi$ and $\xi^{-1}$ (the solid curve in Figure 4 ) on which $\operatorname{Ref}(z)<0$ except at $z=\xi, \xi^{-1}$. Let
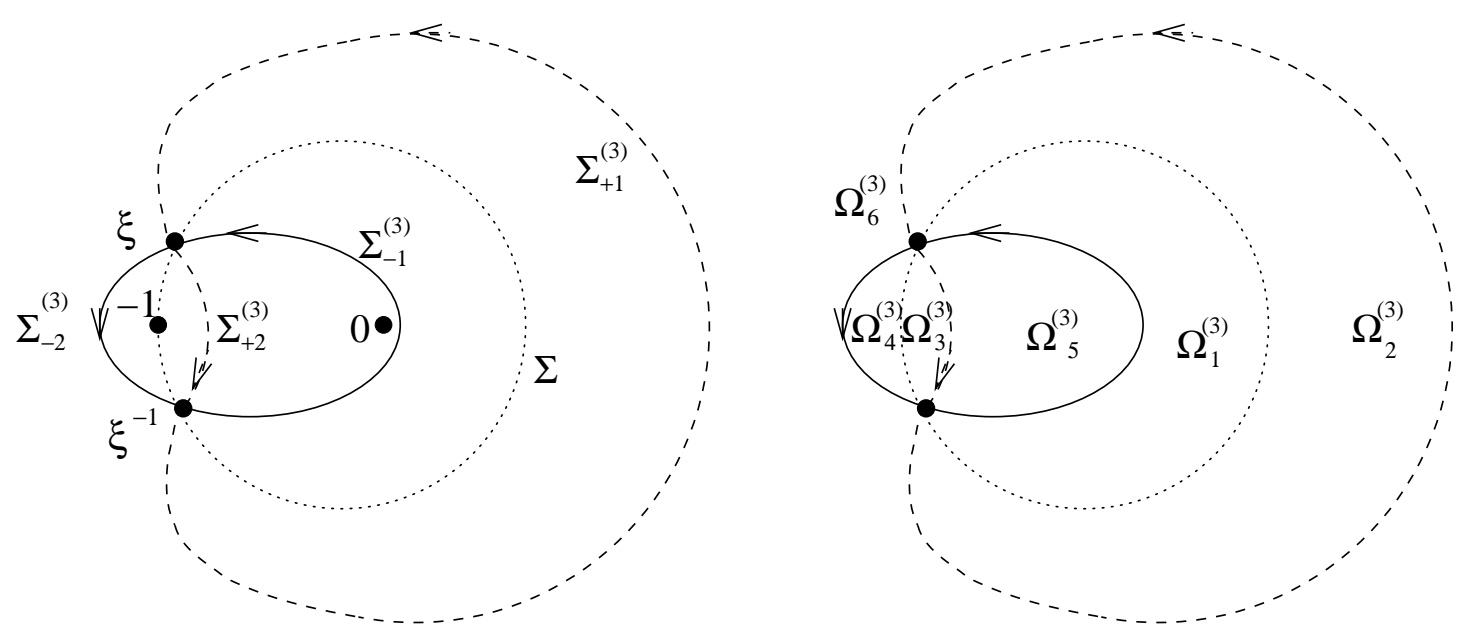

Figure $4: \Sigma_{ \pm}^{(3)}$ and $\Omega_{j}^{(3)}, j=1, \cdots, 6$

$\Sigma_{-}^{(3)}=\overline{\Sigma_{-1}^{(3)} \cup \Sigma_{-2}^{(3)}}$ where $\Sigma_{-1}^{(3)}$ is the open subset of $\Sigma_{-}^{(3)}$ satisfying $|\arg (z)|<\theta_{c}$ and $\Sigma_{-2}^{(3)}=\Sigma_{-}^{(3)} \backslash \overline{\Sigma_{-1}^{(3)}}$. Similarly, we can take an oriented closed curve $\Sigma_{+}^{(3)}$ surrounding 0 , but not -1 and passing through $\xi, \xi^{-1}$ (the dashed curve in Figure 1 ) on which $\operatorname{Re} f(z)>0$ except at $z=\xi, \xi^{-1}$. Again let $\Sigma_{+}^{(3)}=\overline{\Sigma_{+1}^{(3)} \cup \Sigma_{+2}^{(3)}}$ where $\Sigma_{+1}^{(3)}$ is the open subset of $\Sigma_{+}^{(3)}$ satisfying $|\arg (z)|<\theta_{c}$ and $\Sigma_{+2}^{(3)}=\Sigma_{+}^{(3)} \backslash \overline{\Sigma_{+1}^{(3)}}$. The shape of $\Sigma_{ \pm}^{(3)}$ will be specified further below (see the third case for the estimation of $\left|v_{R}(z)-I\right|$ between (5.88) and 
(5.89)). Let $\Sigma^{(3)}=\overline{\Sigma_{-}^{(3)} \cup \Sigma_{+}^{(3)}}$. Let $\Omega_{j}^{(3)}, 1 \leq j \leq 6$ be open regions as in Figure 6 . Define

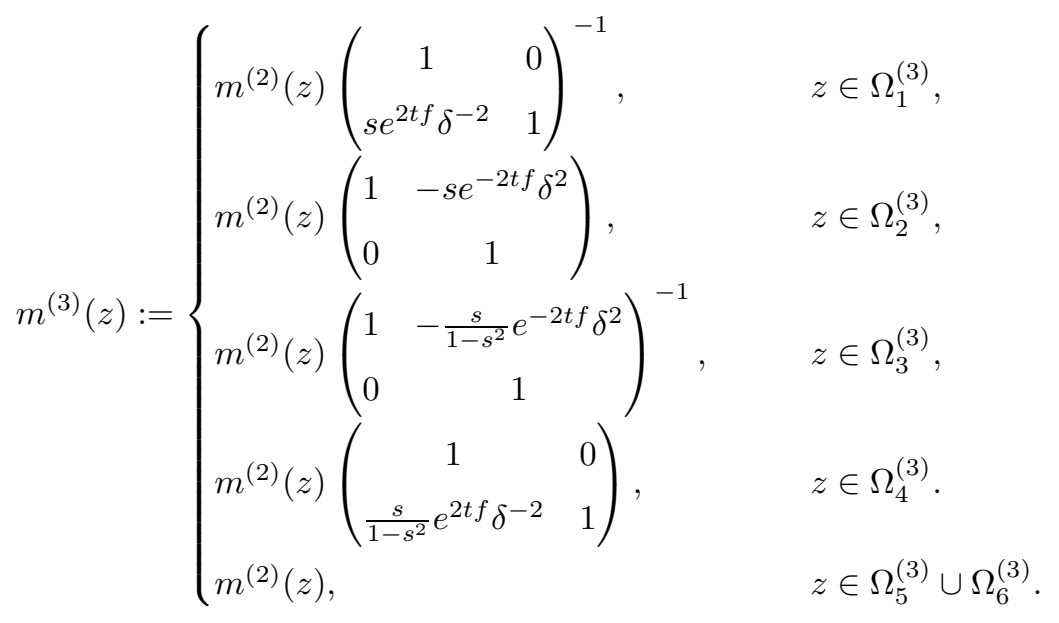

Then (i) $m^{(3)}(z)$ is analytic in $\mathbb{C} \backslash \Sigma^{(3)}$, (ii) $m^{(3)}(z) \rightarrow I$ as $z \rightarrow \infty$, and $m_{+}^{(3)}(z)=m_{-}^{(3)}(z) v^{(3)}$ for $z \in \Sigma^{(3)}$, where

$$
v^{(3)}(z)= \begin{cases}\left(\begin{array}{cc}
1 & 0 \\
s e^{2 t f} \delta^{-2} & 1
\end{array}\right), & z \in \Sigma_{-1}^{(3)}, \\
\left(\begin{array}{cc}
1 & -s e^{-2 t f} \delta^{2} \\
0 & 1
\end{array}\right), & z \in \Sigma_{+1}^{(3)}, \\
\left(\begin{array}{cc}
1 & -\frac{s}{1-s^{2}} e^{-2 t f} \delta^{2} \\
0 & 1
\end{array}\right), & z \in \Sigma_{+2}^{(3)}, \\
\left(\begin{array}{cc}
1 & 0 \\
\frac{s}{1-s^{2}} e^{2 t f} \delta^{-2} & 1
\end{array}\right), & z \in \Sigma_{-2}^{(3)} .\end{cases}
$$

Also we have

$$
m_{11}(0)=m_{11}^{(3)}(0)\left(1-s^{2}\right)^{1-\frac{\theta_{c}}{\pi}} .
$$

Observe that $v^{(3)}(z) \rightarrow I$ as $t \rightarrow \infty$ for $z \in \Sigma^{(3)} \backslash\left\{\xi, \xi^{-1}\right\}$. Thus we expect that $m^{(3)}(z) \rightarrow I$ as $t \rightarrow \infty$. If this were indeed true, we would have

$$
\log m_{11}(0) \sim \frac{\sin ^{-1} \sqrt{1-\eta^{2}}}{\pi} \log \left(1-s^{2}\right) \leq \frac{\log \left(1-s^{2}\right)}{\pi} \sqrt{1-\left(\frac{k}{2 t}\right)^{2}}, \quad t \rightarrow \infty .
$$

But the difficulty, however, is that $v^{(3)}$ does not converge to $I$ uniformly on $\Sigma^{(3)}$. As in [16], we overcome this difficulty by constructing a parametrix for the solution of the $\operatorname{RHP}\left(\Sigma^{(3)}, v^{(3)}\right)$ around the points $\xi, \xi^{-1}$.

Let $\tau$ be a complex number satisfying $0<|\tau|<1$. Following [16], set

$$
\nu:=-\frac{1}{2 \pi} \log \left(1-|\tau|^{2}\right), \quad a:=i \nu .
$$


Define

$$
\beta_{12}:=\frac{\sqrt{2 \pi} e^{\frac{\pi}{4} i} e^{-\frac{\pi}{2} \nu}}{\tau \Gamma(-a)}, \quad \beta_{21}:=\overline{\beta_{12}}=\frac{\sqrt{2 \pi} e^{-\frac{\pi}{4} i} e^{-\frac{\pi}{2} \nu}}{\bar{\tau} \Gamma(a)}
$$

Note that

$$
\beta_{12} \beta_{21}=\nu,
$$

as $|\Gamma(i v)|^{2}=\frac{\pi}{\nu \sinh (\pi \nu)}$ for real $\nu \neq 0$. Let $D_{a}$ be the parabolic-cylinder function (see, e.g. 1, 37) which solves

$$
\frac{d^{2}}{d \zeta^{2}} D_{a}(\zeta)+\left(\frac{1}{2}-\frac{\zeta^{2}}{4}+a\right) D_{a}(\zeta)=0
$$

We note that $D_{a}(\zeta)$ is an entire function.

Let the matrix

$$
\Psi(w)=\left(\begin{array}{ll}
\Psi_{11}(w) & \Psi_{12}(w) \\
\Psi_{21}(w) & \Psi_{22}(w)
\end{array}\right), \quad w \in \mathbb{C} \backslash \mathbb{R},
$$

be defined as follows (see [16] Section4) : for $\operatorname{Im}(w)>0$,

$$
\begin{aligned}
& \Psi_{11}(w):=e^{-\frac{3}{4} \pi \nu} D_{a}\left(e^{-\frac{3}{4} \pi i} w\right) \\
& \Psi_{12}(w):=\left(\beta_{21}\right)^{-1} e^{\frac{1}{4} \pi \nu}\left(\frac{d}{d w} D_{-a}\left(e^{-\frac{1}{4} \pi i} w\right)-\frac{i w}{2} D_{-a}\left(e^{-\frac{1}{4} \pi i} w\right)\right) \\
& \Psi_{21}(w):=\left(\beta_{12}\right)^{-1} e^{-\frac{3}{4} \pi \nu}\left(\frac{d}{d w} D_{a}\left(e^{-\frac{3}{4} \pi i} w\right)+\frac{i w}{2} D_{a}\left(e^{-\frac{3}{4} \pi i} w\right)\right) \\
& \Psi_{22}(w):=e^{\frac{1}{4} \pi \nu} D_{-a}\left(e^{-\frac{1}{4} \pi i} w\right)
\end{aligned}
$$

and for $\operatorname{Im}(w)<0$,

$$
\begin{aligned}
& \Psi_{11}(w):=e^{\frac{1}{4} \pi \nu} D_{a}\left(e^{\frac{1}{4} \pi i} w\right) \\
& \Psi_{12}(w):=\left(\beta_{21}\right)^{-1} e^{-\frac{3}{4} \pi \nu}\left(\frac{d}{d w} D_{-a}\left(e^{\frac{3}{4} \pi i} w\right)-\frac{i w}{2} D_{-a}\left(e^{\frac{3}{4} \pi i} w\right)\right) \\
& \Psi_{21}(w):=\left(\beta_{12}\right)^{-1} e^{\frac{1}{4} \pi \nu}\left(\frac{d}{d w} D_{a}\left(e^{\frac{1}{4} \pi i} w\right)+\frac{i w}{2} D_{a}\left(e^{\frac{1}{4} \pi i} w\right)\right), \\
& \Psi_{22}(w):=e^{-\frac{3}{4} \pi \nu} D_{-a}\left(e^{\frac{3}{4} \pi i} w\right),
\end{aligned}
$$

The function $\Psi$ satisfies

- $\Psi(w)$ is analytic in $w \in \mathbb{C} \backslash \mathbb{R}$.

- For $w \in \mathbb{R}$,

$$
\Psi_{+}(w)=\Psi_{-}(w)\left(\begin{array}{cc}
1-|\tau|^{2} & -\bar{\tau} \\
\tau & 1
\end{array}\right)
$$

where $\Psi_{+}(w)$ (resp., $\left.\Psi_{-}(w)\right)$ is the limit of $\Psi(s)$ as $s \rightarrow w$ with $\operatorname{Im}(s)>0$ (resp., $\left.\operatorname{Im}(s)<0\right)$. 
- As $w \rightarrow \infty$,

$$
\Psi(w) e^{\frac{1}{4} i w^{2} \sigma_{3}} w^{-i \nu \sigma_{3}}=I+O\left(w^{-1}\right)
$$

where $w^{-i \nu}$ denotes the branch which is analytic in $\mathbb{C} \backslash(-\infty, 0]$ and has modulus 1 for $w \in(0, \infty)$.

These properties can be found in [16] Section 4 .

Let $\Gamma$ be the union of four rays, labeled by $\Gamma_{j}, j=1, \cdots, 4$, with the orientation as indicated in Figure 5 . All the rays and $\mathbb{R}$ meet at the angle $\pi / 3$. Denote the components of $\mathbb{C} \backslash(\Gamma \cup \mathbb{R})$ by $\Omega_{j}$,

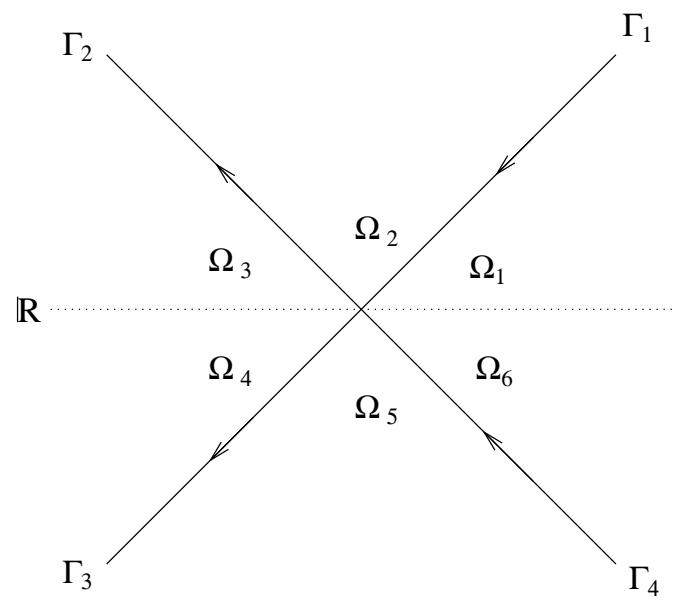

Figure 5: $\Gamma_{j}$ and $\Omega_{j}$

$j=1, \cdots, 6$ as in Figure 5 . Define $H(w)$, analytic in $\mathbb{C} \backslash \Gamma$, by

$$
H(w):=\left(\begin{array}{cc}
a(\xi)^{-1} & 0 \\
0 & a(\xi)
\end{array}\right)\left(\begin{array}{ll}
0 & 1 \\
1 & 0
\end{array}\right) \Psi(w) e^{\frac{1}{4} i w^{2} \sigma_{3}} w^{-i \nu \sigma_{3}}\left(\begin{array}{cc}
0 & 1 \\
1 & 0
\end{array}\right) \phi(w)\left(\begin{array}{cc}
a(\xi) & 0 \\
0 & a(\xi)^{-1}
\end{array}\right),
$$

where $a(\xi)$ is

$$
a(\xi)=e^{t f(\xi)}\left(\frac{-i \xi}{\left(\xi-\xi^{-1}\right) \sqrt{2 t}\left(1-\eta^{2}\right)^{1 / 4}}\right)^{i \nu},
$$


and $\phi(w)$ is defined by

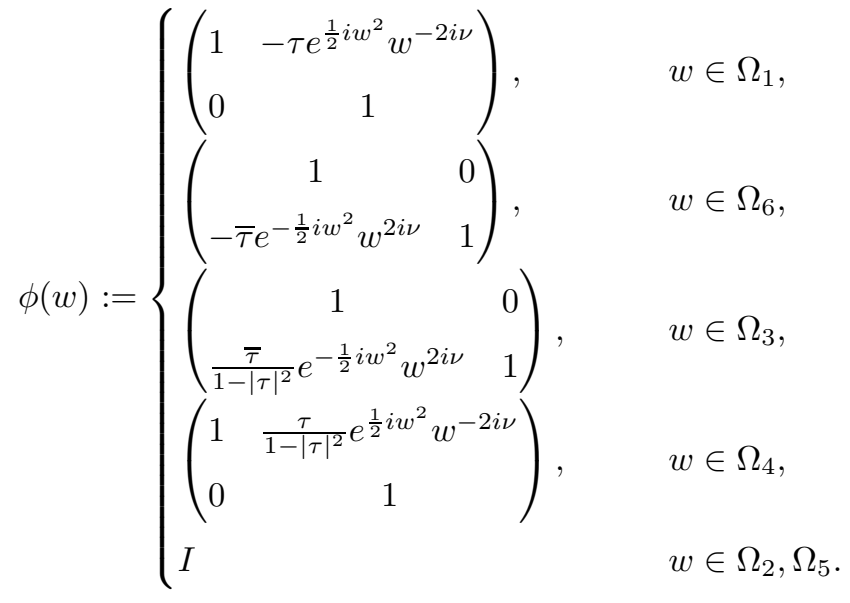

Then by recalling that $w^{-i \nu \sigma_{3}}$ is analytic in $\mathbb{C} \backslash(-\infty, 0]$, one can directly check that $H_{+}(w)=$ $H_{-}(w) v_{H}(w)$ for $w \in \Gamma$, where $v_{H}(w)$ is given by

$$
v_{H}(w):= \begin{cases}\left(\begin{array}{cc}
1 & -\tau a(\xi)^{-2} w^{-2 i \nu} e^{\frac{1}{2} i w^{2}} \\
0 & 1
\end{array}\right), & w \in \Gamma_{1}, \\
\left(\begin{array}{cr}
1 & 0 \\
\bar{\tau} a(\xi)^{2} w^{2 i \nu} e^{-\frac{1}{2} i w^{2}} & 1
\end{array}\right), & w \in \Gamma_{4}, \\
1 & \\
\left(\begin{array}{cr}
\frac{\bar{\tau}}{1-|\tau|^{2}} a(\xi)^{2} w^{2 i \nu} e^{-\frac{1}{2} i w^{2}} & 1
\end{array}\right), & w \in \Gamma_{2}, \\
\left(\begin{array}{cc}
1 & \frac{-\tau}{1-\left.\tau\right|^{2}} a(\xi)^{-2} w^{-2 i \nu} e^{\frac{1}{2} i w^{2}} \\
0 & 1
\end{array}\right), & w \in \Gamma_{3} .\end{cases}
$$

Also, from (5.64), we have

$$
H(w)=I+O\left(w^{-1}\right), \quad \text { as } w \rightarrow \infty .
$$

As $|a(\xi)|=e^{-\nu \theta_{c}},-\pi / 2<\theta_{c}<\pi$, we see that the error term $O\left(w^{-1}\right)$ in (5.69) is uniform for $\frac{2 t}{k}>1$. Similarly, $|H(w)|$ is uniformly bounded in the $w$ plane for $\frac{2 t}{k}>1$.

Define the map

$$
z \mapsto w(z):=\sqrt{2 t}\left(1-\eta^{2}\right)^{1 / 4} i \xi^{-1}(z-\xi)
$$

It maps $\xi$ to 0 , and the tangent line $L_{\xi}$ to the unit circle $\Sigma$ at $\xi$, to the real line as in Figure 6. Let $\mathcal{O}_{\xi}$, $\mathcal{O}_{\bar{\xi}}$ be the disjoint sets $\{z:|z-\xi|<\varrho\},\{z:|z-\bar{\xi}|<\varrho\}$, respectively, where $\varrho$ is defined by

$$
\varrho= \begin{cases}\frac{\epsilon}{2}|\xi-\bar{\xi}|=\epsilon \sqrt{1-\eta^{2}}, & 1+\frac{M_{0}}{2^{1 / 3} k^{2 / 3}} \leq \frac{2 t}{k}<1+\delta, \\ \epsilon, & 1+\delta \leq \frac{2 t}{k} .\end{cases}
$$




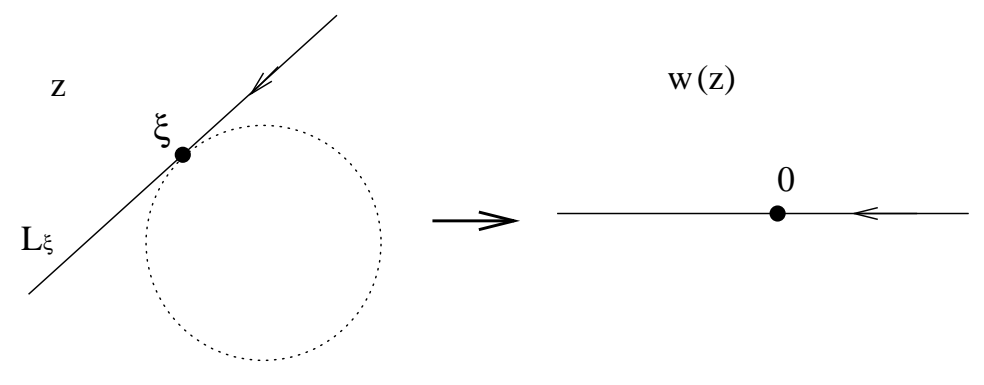

Figure 6: map $z \mapsto w(z)$

The (small) parameter $0<\epsilon<1$ will be specified below (see (5.105) below). We note that one may choose the curves in $\Sigma^{(3)}$ above so that in $\mathcal{O}_{\xi}, \mathcal{O}_{\bar{\xi}}$, they are straight lines which map under $z \mapsto w(z)$ to (finite subsets of ) the rays $\Gamma_{j}, j=1, \cdots, 4, \Sigma_{-1}^{(3)} \cap \mathcal{O}_{\xi} \rightarrow \Gamma_{4}, \Sigma_{-2}^{(3)} \cap \mathcal{O}_{\xi} \rightarrow \Gamma_{2}, \Sigma_{+1}^{(3)} \cap \mathcal{O}_{\xi} \rightarrow \Gamma_{1}$, $\Sigma_{+2}^{(3)} \cap \mathcal{O}_{\xi} \rightarrow \Gamma_{3}$, and similarly for the neighborhood of $\mathcal{O}_{\bar{\xi}}$. For $\tau=s$, we define

$$
m_{p}(z):= \begin{cases}H(w(z)), & z \in \mathcal{O}_{\xi} \backslash \Sigma^{(3)}, \\ \overline{H(w(\bar{z}))}, & z \in \mathcal{O}_{\bar{\xi}} \backslash \Sigma^{(3)}, \\ I, & z \in \mathbb{C} \backslash \overline{\left(\mathcal{O}_{\xi} \cup \mathcal{O}_{\bar{\xi}}\right)}\end{cases}
$$

Let $\Sigma_{R}:=\Sigma^{(3)} \cup \partial \mathcal{O}_{\xi} \cup \partial \mathcal{O}_{\bar{\xi}}$ as in Figure 7 where $\partial \mathcal{O}_{\xi}$ and $\mathcal{O}_{\bar{\xi}}$ are oriented counterclockwise. Clearly $m_{p}$

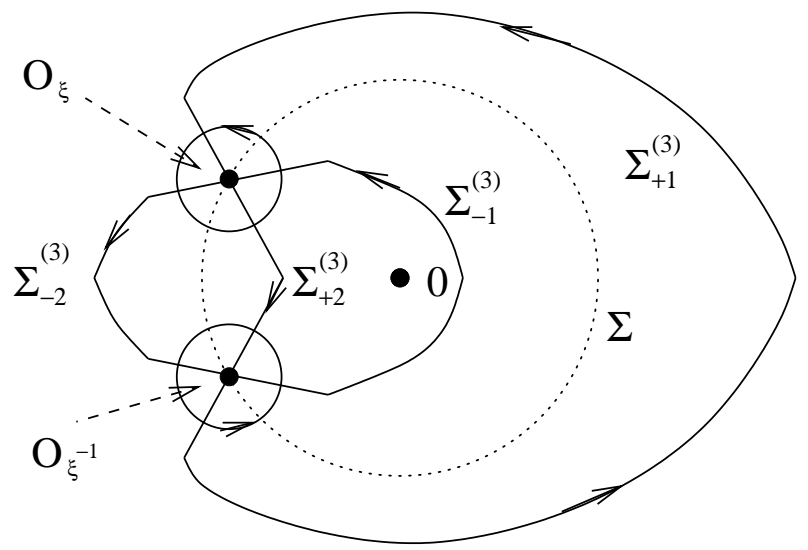

Figure 7: $\Sigma_{R}:=\Sigma^{(3)} \cup \partial \mathcal{O}_{\xi} \cup \partial \mathcal{O}_{\bar{\xi}}$

solves a RHP on $\Sigma_{R}: m_{p}(z)$ is analytic in $\mathbb{C} \backslash \Sigma^{(3)}, m_{p}(z) \rightarrow I$ as $z \rightarrow \infty$, and $m_{p+}(z)=m_{p-}(z) v_{p}(z)$ for $z \in \Sigma_{R}$ for a suitable jump matrix $v_{p}$. Set $R(z):=m^{(3)}(z) m_{p}(z)^{-1}$. Then $R_{+}(z)=R_{-}(z) v_{R}(z)$ for $z \in \Sigma_{R}$ where $v_{R}=m_{p-} v^{(3)} v_{p}^{-1} m_{p-}^{-1}$. Now we estimate $\left|v_{R}(z)-I\right|$. 
- For $z \in \partial \mathcal{O}_{\xi}$, we have from (5.71), when $1+\frac{M_{0}}{2^{1 / 3} k^{2 / 3}} \leq \frac{2 t}{k} \leq 1+\delta$,

$$
\begin{aligned}
|w(z)| & =\epsilon\left(1-\eta^{2}\right)^{3 / 4} \sqrt{2 t} \\
& \geq \epsilon\left(\frac{2 t}{k}\right)^{-1 / 4}\left(k^{2 / 3}\left(\frac{2 t}{k}-1\right)\right)^{3 / 4} \\
& \geq \frac{\epsilon M_{0}^{3 / 4}}{2^{1 / 4}(1+\delta)^{1 / 4}} .
\end{aligned}
$$

When $1+\delta \leq \frac{2 t}{k}$

$$
|w(z)|=\sqrt{2 t}\left(1-\eta^{2}\right) \epsilon \geq\left(\frac{\delta}{1+\delta}\right)^{1 / 4} \epsilon \sqrt{2 t} .
$$

Thus if we have taken $M_{0}$ large, and $t$ is large, we have for $z \in \partial \mathcal{O}_{\xi}$, from (5.69),

$$
m_{p+}(z)=I+O\left(\frac{1}{\min \left(M_{0}^{3 / 4}, \sqrt{t}\right)}\right) .
$$

But as $v^{(3)}=I$ on $\partial \mathcal{O}_{\xi}, v_{R}(z)=v_{p}(z)^{-1}=m_{p+}(z)^{-1}$, and hence

$$
\left\|v_{R}(z)-I\right\|_{L^{\infty}\left(\partial \mathcal{O}_{\xi}\right)} \leq \frac{C}{\min \left(M_{0}^{3 / 4}, \sqrt{t}\right)} \quad z \in \partial \mathcal{O}_{\xi} .
$$

We are using here the standard fact that if $\operatorname{det} v_{p}=1$, then $\operatorname{det} m_{p}=1$. Similarly, we have the same estimate (5.76) on $v_{R}(z)$ for $z \in \partial \mathcal{O}_{\bar{\xi}}$.

- For $z \in \Sigma^{(3)} \cap \mathcal{O}_{\xi}$, since $m_{p}$ and $m_{p}^{-1}$ are uniformly bounded, $\left|v_{R}(z)-I\right| \leq C\left|v^{(3)}(z) v_{p}(z)^{-1}-I\right|$. For $z \in \Sigma_{-1}^{(3)} \cap \mathcal{O}_{\xi}$, by (5.47), (5.68),

$$
\begin{aligned}
\left|v^{(3)}(z) v_{p}(z)^{-1}-I\right| & \leq\left|v_{21}^{(3)}(z)-\left(v_{H}\right)_{21}(w(z))\right| \\
& =s\left|e^{2 t f(z)} \delta^{-2}(z)-a(\xi)^{2} w(z)^{2 i \nu} e^{-\frac{1}{2} i w(z)^{2}}\right|=: s|\Delta| .
\end{aligned}
$$

Setting $u:=i \xi^{-1}(z-\xi)$, we have

$$
\begin{aligned}
\Delta= & e^{2 t\left(\frac{1}{2}\left(\xi(1-i u)-\frac{1}{\xi(1-i u)}\right)+\eta \log \xi(1-i u)\right)}\left(\frac{-i \xi u}{\xi(1-i u)-\xi^{-1}}\right)^{2 i \nu} \\
& -e^{2 t\left(\frac{1}{2}\left(\xi-\xi^{-1}\right)+\eta \log \xi\right)-t i\left(1-\eta^{2}\right)^{1 / 2} u^{2}}\left(\frac{-i \xi u}{\xi-\xi^{-1}}\right)^{2 i \nu} \\
= & \left(e^{2 t h(u)} j(u)-1\right) e^{2 t\left(\frac{1}{2}\left(\xi-\xi^{-1}\right)+\eta \log \xi\right)-t i\left(1-\eta^{2}\right)^{1 / 2} u^{2}}\left(\frac{-i \xi u}{\xi-\xi^{-1}}\right)^{2 i \nu}
\end{aligned}
$$

where

$$
\begin{aligned}
h(u) & =\frac{1}{2}\left(-i \xi u-\frac{i u}{\xi(1-i u)}\right)+\eta \log (1-i u)+\frac{1}{2} i\left(1-\eta^{2}\right)^{1 / 2} u^{2} \\
& =\left(-\frac{1}{6} i \eta+\frac{1}{2} \sqrt{1-\eta^{2}}\right) u^{3}+O\left(u^{4}\right),
\end{aligned}
$$


and

$$
j(u)=\left(\frac{\xi-\xi^{-1}}{\xi-\xi^{-1}-i \xi u}\right)^{2 i \nu}=1+O\left(\frac{u}{\xi-\xi^{-1}}\right)=1+O\left(\frac{u}{\sqrt{1-\eta^{2}}}\right) .
$$

Also, as $\left|\frac{u}{\sqrt{1-\eta^{2}}}\right| \leq c \epsilon$ for $z \in \mathcal{O}_{\xi}$, we have

$$
\begin{aligned}
|h(u)| & \leq c|u|^{3} \\
j(u) & =1+O\left(\frac{u}{\sqrt{1-\eta^{2}}}\right)=1+O(\epsilon) .
\end{aligned}
$$

On the other hand, for $z \in \Sigma_{-1}^{(3)} \cap \mathcal{O}_{\xi}$,

$$
\operatorname{Re}\left(-i t \sqrt{1-\eta^{2}} u^{2}\right) \leq-c t \sqrt{1-\eta^{2}}|u|^{2}, \quad c=\cos \frac{\pi}{6}>0 .
$$

Therefore, we obtain

$$
\begin{aligned}
|\Delta| & \leq C\left|\left(e^{2 t h(u)}-1\right)+e^{2 t h(u)}(j(u)-1)\right| e^{-c t \sqrt{1-\eta^{2}}|u|^{2}} \\
& \leq C(|2 t h(u)|+|j(u)-1|) e^{-c t \sqrt{1-\eta^{2}}|u|^{2}+|\operatorname{Re}(2 t h(u))|} \\
& \leq C\left(t|u|^{3}+\epsilon\right) e^{-c t \sqrt{1-\eta^{2}}|u|^{2}} \\
& \leq \frac{C}{\sqrt{2 t}\left(1-\eta^{2}\right)^{3 / 4}}+C \epsilon,
\end{aligned}
$$

where for the last inequality, we have used the fact that $\left|x^{3} e^{-x^{2}}\right|$ is uniformly bounded for $x \in \mathbb{R}$. Now for $1+\frac{M_{0}}{2^{1 / 3} k^{2 / 3}} \leq \frac{2 t}{k}<1+\delta$, we have

$$
\sqrt{2 t}\left(1-\eta^{2}\right)^{3 / 4}>\sqrt{2 t}(1-\eta)^{3 / 4}=\left(\frac{k}{2 t}\right)^{1 / 4}\left(k^{2 / 3}\left(\frac{2 t}{k}-1\right)\right)^{3 / 4} \geq \frac{1}{(1+\delta)^{1 / 4}}\left(\frac{M_{0}}{2^{1 / 3}}\right)^{3 / 4}
$$

and for $1+\delta \leq \frac{2 t}{k}$, we have

$$
\sqrt{2 t}\left(1-\eta^{2}\right)^{3 / 4} \geq \sqrt{2 t}\left(\frac{\delta}{1+\delta}\right)^{3 / 4}
$$

Thus we obtain (recall (5.77))

$$
\left\|v_{R}-I\right\|_{L^{\infty}\left(\Sigma_{-1}^{(3)} \cap \mathcal{O}_{\xi}\right)} \leq C s\|\Delta\|_{L^{\infty}\left(\Sigma_{-1}^{(3)} \cap \mathcal{O}_{\xi}\right)} \leq \frac{C}{\min \left(M_{0}^{3 / 4}, \sqrt{t}\right)}+C \epsilon
$$

which is small if we take $M_{0}, t$ large and $\epsilon$ small. For other parts of $\Sigma^{(3)} \cap \mathcal{O}_{\xi}$, by a similar argument, we obtain the same estimate. By the symmetry $m^{(3)}(z)=\overline{m^{(3)}(\bar{z})}$ and $m_{p}(z)=\overline{m_{p}(\bar{z})}$, we obtain the same estimate for $\Sigma^{(3)} \cap \mathcal{O}_{\bar{\xi}}$.

- Let $\mathcal{O}:=\mathcal{O}_{\xi} \cup \mathcal{O}_{\bar{\xi}}$. For $z \in \Sigma_{-1}^{(3)} \cap(\mathbb{C} \backslash \overline{\mathcal{O}}), v_{R}(z)=v^{(3)}(z)$. Thus we need an estimate for $v_{21}^{(3)}(z)=\operatorname{se}^{2 t f(z)} \delta^{-2}(z)$. Since $|\delta(z)|=e^{-\nu \theta}$ where $\theta=\arg \left(\frac{z-\xi}{z-\bar{\xi}}\right),|\delta(z)|$ and $\left|\delta^{-1}(z)\right|$ are uniformly bounded. When $1+\delta \leq \frac{2 t}{k}, \operatorname{dist}\left(\Sigma_{-1}^{(3)} \cap \overline{\mathcal{O}}^{c},\left\{\xi, \xi^{-1}\right\}\right.$ is uniformly bounded below. From this fact, 
one can check that we can take $\Sigma_{-1}^{(3)}$ so that $\operatorname{Re}(f(z)) \leq-c_{0}(\epsilon)$ for $z \in \Sigma_{-1}^{(3)} \cap \overline{\mathcal{O}}^{c}$ for some constant $c_{0}(\epsilon)>0$ depending on $\epsilon$. Hence we have

$$
\left\|v_{R}-I\right\|_{L^{\infty}\left(\Sigma_{-1}^{(3)} \cap \overline{\mathcal{O}}^{c}\right)} \leq C e^{-c_{0}(\epsilon) t}, \quad 1+\delta \leq \frac{2 t}{k} .
$$

On the other hand, when $1+\frac{M_{0}}{2^{1 / 3} k^{2 / 3}} \leq \frac{2 t}{k}<1+\delta$, we take $\Sigma_{-1}^{(3)}=\left\{\rho(\theta) e^{i \theta}:|\theta|<\theta_{c}\right\}$ such that

(i) For $z \in \Sigma_{-1}^{(3)}$ with $\frac{2 \pi}{3}<|\arg (z)|<\theta_{c}, \Sigma_{-1}^{(3)}$ is a pair of straight lines which meet the unit circle at $\xi$ and $\bar{\xi}$, respectively, with angle $\pi / 3$.

(ii) For $\rho e^{i \theta} \in \Sigma_{-1}^{(3)}$ with $|\arg (z)| \leq \frac{2 \pi}{3}, \operatorname{Re}\left(f\left(\rho e^{i \theta}\right)\right) \leq \operatorname{Re}\left(f\left(\rho^{\prime} e^{i \theta}\right)\right)$ for $\rho \leq \rho^{\prime} \leq 1$. Also $\rho(\theta)$ is an increasing function for $0<\theta<\frac{2 \pi}{3}$ and is a decreasing function for $-\frac{2 \pi}{3}<\theta<0$.

(Here the precise value $\frac{2 \pi}{3}$ is of no importance : any angle between $\pi / 2$ and $\pi$ will do.) Condition (ii) can be achieved by choosing $\Sigma_{-1}^{(3)}$ always to be above the curve $\left\{\rho_{\theta} e^{i \theta}: \frac{\pi}{2}<\theta \leq \theta_{c}\right\}$ (recall (5.38) and Figures 1. 3). Condition (i) can be achieved as the curve $\left\{\rho_{\theta} e^{i \theta}: \frac{\pi}{2}<\theta \leq \theta_{c}\right\}$ crosses the unit circle at 90 degree (see Figure 3) For $z$ in (i) satisfying $\arg (z)>0$, we have $z=\xi\left(1-i r e^{-\frac{\pi}{3} i}\right)$ for some real $r>0$. We note that $r \leq \frac{\sqrt{3}}{2}<\frac{2}{\sqrt{3}}$. For such $z$, we have (recall (5.35)

$$
\operatorname{Re}(f(z))=A(r) \sqrt{1-\eta^{2}}+B(r) \eta,
$$

where

$$
A(r)=\frac{r^{2}(r-\sqrt{3})}{4\left(1-\sqrt{3} r+r^{2}\right)}, \quad B(r)=-\frac{r(r-\sqrt{3})(2-\sqrt{3} r)}{4\left(1-\sqrt{3} r+r^{2}\right)}+\frac{1}{2} \log \left(1-\sqrt{3} r+r^{2}\right) .
$$

One can easily check that $A(r)<0$ for $0<r<\sqrt{3}$ and $B(r)<0$ for $0<r<\frac{2}{\sqrt{3}}$. Thus for $z$ in (i) satisfying $\arg (z)>0$, we have for some $c>0$,

$$
\operatorname{Re}(f(z)) \leq A(r) \sqrt{1-\eta^{2}} \leq-c r^{2} \sqrt{1-\eta^{2}} .
$$

For $z$ in (ii), note first that for fixed $0<\rho<1, \operatorname{Re}\left(f\left(\rho e^{i \theta}\right)\right.$ is an increasing function in $0 \leq \theta<\pi$. Let $z_{b}$ be the point on $\Sigma_{-1}^{(3)}$ satisfying $\arg (z)=\frac{2 \pi}{3}$. Thus together with the condition (ii), we obtain for $z$ in (ii) satisfying $\arg (z)>0$,

$$
\operatorname{Re}(f(z)) \leq \operatorname{Re}\left(f\left(z_{b}\right)\right) \leq-c\left|z_{b}-\xi\right|^{2} \sqrt{1-\eta^{2}} \leq-c|z-\xi|^{2} \sqrt{1-\eta^{2}} .
$$

Here the second inequality follows from (5.91). Thus we have for $z \in \Sigma_{-1}^{(3)} \cap{\overline{\mathcal{O}_{\xi}}}^{c}$ with $\arg (z)>0$,

$$
\left|v_{R}(z)-I\right| \leq \begin{cases}C e^{-c_{0}(\epsilon) t}, & 1+\delta \leq \frac{2 t}{k}, \\ C e^{-c t|z-\xi|^{2} \sqrt{1-\eta^{2}}}, & 1+\frac{M_{0}}{2^{1 / 3} k^{2 / 3}} \leq \frac{2 t}{k}<1+\delta .\end{cases}
$$

By symmetry, we have similar estimates for $z \in \Sigma_{-1}^{(3)}$ with $\arg (z)<0$. Since $|z-\xi|>\varrho$ for $z \in \overline{\mathcal{O}}^{c}$, the above estimates imply in particular that

$$
\left\|v_{R}-I\right\|_{L^{\infty}\left(\Sigma_{-1}^{(3)} \cap(\mathbb{C} \backslash \overline{\mathcal{O}})\right)} \leq \begin{cases}C e^{-c \epsilon^{2} M_{0}^{3 / 2}}, & 1+\frac{M_{0}}{2^{1 / 3} k^{2 / 3}} \leq \frac{2 t}{k}<1+\delta \\ C e^{-c_{0}(\epsilon) t}, & 1+\delta \leq \frac{2 t}{k}\end{cases}
$$


For $\Sigma_{+1}^{(3)} \cap(\mathbb{C} \backslash \overline{\mathcal{O}})$, by the symmetry $\operatorname{Re}\left(f\left(\rho e^{i \theta}\right)\right)=\operatorname{Re}\left(f\left(\rho^{-1} e^{i \theta}\right)\right.$, we have the same estimate. Also by a similar argument, we obtain a similar estimate for $\left(\Sigma_{-2}^{(3)} \cup \Sigma_{+2}^{(3)}\right) \cap(\mathbb{C} \backslash \overline{\mathcal{O}})$.

As usual, define an operator on $L^{2}\left(\Sigma_{R}\right)$,

$$
C_{v_{R}}(f)=C_{-}\left(f\left(v_{R}-I\right)\right)
$$

where $C_{-}$is the Cauchy operator

$$
\left(C_{-} f\right)(z)=\lim _{z^{\prime} \rightarrow z} \frac{1}{2 \pi i} \int_{\Sigma_{R}} \frac{f(s)}{s-z^{\prime}} d s, \quad z \in \Sigma_{R}, \quad z^{\prime} \text { on the }- \text { side of } \Sigma_{R} .
$$

As the Cauchy operator is scale invariant, $C_{-}$is bounded from $L^{2}\left(\Sigma_{R}\right) \rightarrow L^{2}\left(\Sigma_{R}\right)$ uniformly for $\frac{2 t}{k} \geq 1+\frac{M_{0}}{2^{1 / 3} k^{2 / 3}}$, and we have $\left\|C_{v_{R}}\right\|<\frac{1}{2}$ for $t, M_{0}$ sufficiently large by (5.76), (5.87) and (5.94). Hence $1-C_{v_{R}}$ is invertible. By standard facts in Riemann-Hilbert theory (see [11, 6]), the solution $R(z)$ to the $\operatorname{RHP}\left(\Sigma_{R}, v_{R}\right)$ is given by

$$
R(z)=I+\frac{1}{2 \pi i} \int_{\Sigma_{R}} \frac{\left(I+\left(1-C_{v_{R}}\right)^{-1} C_{v_{R}} I\right)\left(v_{R}-I\right)(s)}{s-z} d s .
$$

As $m_{p}(0)=I$, we have $m_{11}^{(3)}(0)=R_{11}(0)$. By using $\operatorname{dist}\left(0, \Sigma_{R}\right)>0,\left\|\left(1-C_{v_{R}}\right)^{-1}\right\| \leq c$, and $\left\|C_{-}\right\| \leq c$, we have

$$
\begin{aligned}
\left|m_{11}^{(3)}(0)-1\right| & \leq c\left\|v_{R}-I\right\|_{L^{1}\left(\Sigma_{R}\right)}+c\left\|\left(1-C_{v_{R}}\right)^{-1} C_{v_{R}} I\right\|_{L^{2}\left(\Sigma_{R}\right)}\left\|v_{R}-I\right\|_{L^{2}\left(\Sigma_{R}\right)} \\
& \leq c\left\|v_{R}-I\right\|_{L^{1}}+c\left\|\left(1-C_{v_{R}}\right)^{-1}\right\|_{L^{2} \rightarrow L^{2}}\left\|C_{-}\left(v_{R}-I\right)\right\|\left\|_{L^{2}}\right\| v_{R}-I \|_{L^{2}} \\
& \leq c\left\|v_{R}-I\right\|_{L^{1}}+c\left\|v_{R}-I\right\|_{L^{2}}^{2} \\
& \leq c\left\|v_{R}-I\right\|_{L^{1}}+c\left\|v_{R}-I\right\|_{L^{\infty}}\left\|v_{R}-I\right\|_{L^{1}} \\
& \leq c\left\|v_{R}-I\right\|_{L^{1}\left(\Sigma_{R}\right)}
\end{aligned}
$$

as $\left\|v_{R}-I\right\|_{L^{\infty}}$ is bounded. We estimate $\left\|v_{R}-I\right\|_{L^{1}}$ in each part of $\Sigma_{R}$. First, for $\partial \mathcal{O}$ and $\Sigma^{(3)} \cap \mathcal{O}$, since the length of the contour is of order $\varrho$, we obtain by (5.76), (5.87)

$$
\left\|v_{R}-I\right\|_{L^{1}\left(\Sigma_{R} \cap \overline{\mathcal{O}}\right)} \leq C \varrho\left(\frac{1}{\min \left(M_{0}^{3 / 4}, \sqrt{t}\right)}+\epsilon\right) .
$$

When $1+\frac{M_{0}}{2^{1 / 3} k^{2 / 3}} \leq \frac{2 t}{k}<1+\delta$, by (5.71), $\varrho=\epsilon \sqrt{1-\eta^{2}}$. When $1+\delta \leq \frac{2 t}{k}, \sqrt{1-\eta^{2}}=\sqrt{1-(k /(2 t))^{2}} \geq$ $C$, and hence we have $\varrho=\epsilon \leq c \sqrt{1-\eta^{2}}$. Thus in both cases, we obtain

$$
\left\|v_{R}-I\right\|_{L^{1}\left(\Sigma_{R} \cap \overline{\mathcal{O}}\right)} \leq C \sqrt{1-\eta^{2}}\left(\frac{1}{\min \left(M_{0}^{3 / 4}, \sqrt{t}\right)}+\epsilon\right) .
$$

Now we compute $\left\|v_{R}-I\right\|_{L^{1}\left(\Sigma_{R} \cap \overline{\mathcal{O}}^{c}\right)}$. We first focus on $\Sigma_{-1}^{(3)} \cap \overline{\mathcal{O}}^{c} \cap\{\operatorname{Im}(z)>0\}$ When $1+\delta \leq \frac{2 t}{k}$, by (5.93),

$$
\left\|v_{R}-I\right\|_{L^{1}\left(\Sigma_{-1}^{(3)} \cap \overline{\mathcal{O}}^{c} \cap\{\operatorname{Im}(z)>0\}\right)} \leq C e^{-c_{0}(\epsilon) t} \leq \frac{C}{\sqrt{t}} \sqrt{1-\eta^{2}}
$$


for large $t$ as $\sqrt{1-\eta^{2}} \geq C$ in this case. When $1+\frac{M_{0}}{2^{1 / 3} k^{2 / 3}} \leq \frac{2 t}{k}<1+\delta$, from (5.93),

$$
\begin{aligned}
\left\|v_{R}-I\right\|_{L^{1}\left(\Sigma_{-1}^{(3)} \cap \overline{\mathcal{O}}^{c} \cap\{\operatorname{Im}(z)>0\}\right)} & \leq \int_{\Sigma_{-1}^{(3)} \cap \overline{\mathcal{O}}^{c} \cap\{\operatorname{Im}(z)>0\}} C e^{-c t \sqrt{1-\eta^{2}}|z-\xi|^{2}}|d z| \\
& \leq C \int_{\varrho}^{\infty} e^{-c t \sqrt{1-\eta^{2}} r^{2}} d r \\
& \leq \frac{C}{\left(t \sqrt{1-\eta^{2}}\right)^{1 / 2}} e^{-c t \sqrt{1-\eta^{2}} \varrho^{2}} .
\end{aligned}
$$

But since, for $1+\frac{M_{0}}{2^{1 / 3} k^{2 / 3}} \leq \frac{2 t}{k}<1+\delta$,

$$
\sqrt{1-\eta^{2}} \geq \sqrt{1-\frac{k}{2 t}}=\frac{1}{(2 t)^{1 / 3}}\left(\frac{k}{2 t}\right)^{1 / 6} \sqrt{k^{2 / 3}\left(\frac{2 t}{k}-1\right)} \geq \frac{C M_{0}^{1 / 3}}{t^{1 / 3}}
$$

we obtain

$$
\left\|v_{R}-I\right\|_{L^{1}\left(\Sigma_{-1}^{(3)} \cap \overline{\mathcal{O}}^{c} \cap\{\operatorname{Im}(z)>0\}\right)} \leq \frac{C}{M_{0}^{3 / 4}} e^{-c \epsilon^{2} M_{0}^{3 / 2}} \sqrt{1-\eta^{2}} .
$$

By a similar computation, we obtain the same estimate for the other parts of $\Sigma_{R} \cap \overline{\mathcal{O}}^{c}$. Thus if we take $\epsilon$ small, and then take $M_{0}, t$ large, we obtain by (5.98), (5.99) and (5.104),

$$
\left\|m_{11}^{(3)}-1\right\|_{L^{1}\left(\Sigma_{R}\right)} \leq \alpha \sqrt{1-\eta^{2}}
$$

with a constant $\alpha>0$ which can be taken to be arbitrarily small. Therefore, from (5.48), (5.98), using (5.105), we obtain (note (5.49) ) for large $t$,

$$
\log m_{11}(0)=\log m_{11}^{(3)}(0)+\left(1-\frac{\theta_{c}}{\pi}\right) \log \left(1-s^{2}\right) \leq \alpha \sqrt{1-\eta^{2}}-c \sqrt{1-\eta^{2}} \leq-C \sqrt{1-\eta^{2}}
$$

for some $C>0$, which is $(5.18$ ).

\section{Multi-Painlevè Functions}

In this section we will show that the multi-interval case considered in Theorem 2.12 is related to new classes of "multi-Painlevé function". As we will see, these functions describe the interaction of solutions of Painlevé equations in a way which is strongly reminiscent of the interaction of classical solitons. We suggest the name "Painlevétons" or simply "P-tons" for these functions. In this section we only illustrate a few of the properties of P-tons. The general theory will be developed in a subsequent paper together with Alexander Its.

From Theorem 2.12, in the $k$ interval case,

$$
\sum_{j=1}^{k}\left(s_{j}-s_{j-1}\right) \mathrm{K}_{n_{j}}(z, w)=\frac{\sum_{l=0}^{k} f_{l}(z) g_{l}(w)}{z-w}
$$


where

$$
\begin{aligned}
& f=\left(f_{0}, \cdots, f_{k}\right)^{T}=\left(s_{k},\left(s_{1}-s_{0}\right) \varphi(z) z^{n_{1}}, \cdots,\left(s_{k}-s_{k-1}\right) \varphi(z) z^{n_{k}}\right)^{T}, \\
& g=\left(g_{0}, \cdots, g_{k}\right)^{T}=(2 \pi i)^{-1}\left(1,-\left(\varphi(z) z^{n_{1}}\right)^{-1}, \cdots,-\left(\varphi(z) z^{n_{k}}\right)^{-1}\right)^{T} .
\end{aligned}
$$

Thus by the integrable operator theory [21, 12], the associated jump matrix $v$ on $\Sigma=\{|z|=1\}$ has the form

$$
v=I-2 \pi i f g^{T}=\left(\begin{array}{ccc}
1-s_{k} & s_{k}\left(\varphi z^{n_{1}}\right)^{-1} & s_{k}\left(\varphi z^{n_{k}}\right)^{-1} \\
-\left(s_{1}-s_{0}\right) \varphi z^{n_{1}} & \ldots \ldots \ldots & \\
\vdots & \left(\delta_{p q}+\left(s_{p}-s_{p-1}\right) z^{n_{p}-n_{q}}\right.
\end{array}\right)_{1 \leq p, q \leq k}
$$

For purposes of illustration, we will only consider the case when $k=2$,

$$
v=v^{(3)}=\left(\begin{array}{ccc}
1-s_{2} & s_{2}\left(\varphi z^{n_{1}}\right)^{-1} & s_{2}\left(\varphi z^{n_{2}}\right)^{-1} \\
-s_{1} \varphi z^{n_{1}} & 1+s_{1} & s_{1} z^{n_{1}-n_{2}} \\
-\left(s_{2}-s_{1}\right) \varphi z^{n_{2}} & \left(s_{2}-s_{1}\right) z^{n_{2}-n_{1}} & 1+s_{2}-s_{1}
\end{array}\right)
$$

and $\varphi=e^{t\left(z-z^{-1}\right)}$ as in Introduction. Observe now that when $s_{1}=0$, the jump matrix takes the form

$$
v=v^{(3)}=\left(\begin{array}{ccc}
1-s_{2} & s_{2}\left(\varphi z^{n_{1}}\right)^{-1} & s_{2}\left(\varphi z^{n_{2}}\right)^{-1} \\
0 & 1 & 0 \\
-s_{2} \varphi z^{n_{2}} & s_{2} z^{n_{2}-n_{1}} & 1+s_{2}
\end{array}\right) .
$$

Let $m^{(3)}$ be the solution of the $3 \times 3$ RHP

$$
\left\{\begin{array}{l}
m_{+}^{(3)}=m_{-}^{(3)} v^{(3)}, \quad z \in \Sigma, \\
m^{(3)} \rightarrow I \quad \text { as } z \rightarrow \infty
\end{array}\right.
$$

But it is clear that the $2 \times 2$ matrix $m^{(2)}$ constructed from $m^{(3)}$ as follows,

$$
m^{(2)}=\left(\begin{array}{ll}
m_{11}^{(3)} & m_{13}^{(3)} \\
m_{31}^{(3)} & m_{33}^{(3)}
\end{array}\right)
$$

solves the RHP

$$
\left\{\begin{array}{l}
m_{+}^{(2)}=m_{-}^{(2)}\left(\begin{array}{cc}
1-s_{2} & s_{2}\left(\varphi z^{n_{2}}\right)^{-1} \\
-s_{2} \varphi z^{n_{2}} & 1+s_{2}
\end{array}\right), \quad z \in \Sigma, \\
m^{(2)} \rightarrow I \quad \text { as } z \rightarrow \infty
\end{array}\right.
$$


which is an RHP which is algebraically equivalent to the RHP for Painlevé III (PIII) which occurred in [3] : set

$$
\left\{\begin{array}{cc}
\widetilde{m}^{(2)}=\left(\begin{array}{cc}
\sqrt{1+s_{2}} & 0 \\
0 & \frac{1}{\sqrt{1+s_{2}}}
\end{array}\right) m^{(2)}\left(\begin{array}{cc}
\sqrt{1+s_{2}} & 0 \\
0 & \frac{1}{\sqrt{1+s_{2}}}
\end{array}\right) & |z|<1, \\
\widetilde{m}^{(2)}=\left(\begin{array}{cc}
\sqrt{1+s_{2}} & 0 \\
0 & \frac{1}{\sqrt{1+s_{2}}}
\end{array}\right) m^{(2)}\left(\begin{array}{cc}
\frac{1}{\sqrt{1+s_{2}}} & 0 \\
0 & \sqrt{1+s_{2}}
\end{array}\right) & |z|>1 .
\end{array}\right.
$$

Then $\widetilde{m}^{(2)}$ solves the RHP

$$
\left\{\begin{array}{l}
\widetilde{m}_{+}^{(2)}=\widetilde{m}_{-}^{(2)}\left(\begin{array}{cc}
1-s_{2}^{2} & s_{2}\left(\varphi z^{n_{2}}\right)^{-1} \\
-s_{2} \varphi z^{n_{2}} & 1
\end{array}\right), \quad z \in \Sigma, \\
m^{(2)} \rightarrow I \quad \text { as } z \rightarrow \infty
\end{array}\right.
$$

which is the RHP for PIII considered in [18. On the other hand, if $s_{1}=s_{2}=s$, then

$$
v=v^{(3)}=\left(\begin{array}{ccc}
1-s & s\left(\varphi z^{n_{1}}\right)^{-1} & s\left(\varphi z^{n_{2}}\right)^{-1} \\
-s \varphi z^{n_{1}} & 1+s & s z^{n_{1}-n_{2}} \\
0 & 0 & 1
\end{array}\right) .
$$

Now

$$
m^{(2)}=\left(\begin{array}{ll}
m_{11}^{(3)} & m_{12}^{(3)} \\
m_{21}^{(3)} & m_{22}^{(3)}
\end{array}\right)
$$

solves the RHP

$$
\left\{\begin{array}{l}
m_{+}^{(2)}=m_{-}^{(2)}\left(\begin{array}{cc}
1-s & s\left(\varphi z^{n_{2}}\right)^{-1} \\
-s \varphi z^{n_{2}} & 1+s
\end{array}\right), \quad z \in \Sigma, \\
m^{(2)} \rightarrow I \quad \text { as } z \rightarrow \infty
\end{array}\right.
$$

which again is the (equivalent) RHP for PIII. Also if we set $n_{1}=n_{2}=n$,

$$
v=v^{(3)}=\left(\begin{array}{ccc}
1-s_{2} & s_{2}\left(\varphi z^{n}\right)^{-1} & s_{2}\left(\varphi z^{n}\right)^{-1} \\
-s_{1} \varphi z^{n} & 1+s_{1} & s_{1} \\
-\left(s_{2}-s_{1}\right) \varphi z^{n} & \left(s_{2}-s_{1}\right) & 1+s_{2}-s_{1}
\end{array}\right) .
$$

Conjugating the solution $m^{(3)}$ of the RHP associated with $v^{(3)}$ by

$$
m^{(3)} \mapsto \widetilde{m}^{(3)}=\left(\begin{array}{ccc}
1 & 0 & 0 \\
0 & 1 & 0 \\
0 & 1 & 1
\end{array}\right) m^{(3)}\left(\begin{array}{ccc}
1 & 0 & 0 \\
0 & 1 & 0 \\
0 & 1 & 1
\end{array}\right)^{-1}
$$


we find that $\widetilde{m}^{(3)} \rightarrow I$ as $z \rightarrow \infty$, and $\widetilde{m}^{(3)}$ solves a RHP with jump matrix

$$
\widetilde{v}^{(3)}=\left(\begin{array}{ccc}
1-s_{2} & 0 & s_{2}\left(\varphi z^{n}\right)^{-1} \\
-s_{1} \varphi z^{n} & 1 & s_{1} \\
-s_{2} \varphi z^{n} & 0 & 1+s_{2}
\end{array}\right)
$$

It follows that necessarily

$$
\left(\begin{array}{lll}
\widetilde{m}_{12}^{(3)} & \widetilde{m}_{22}^{(3)} & \widetilde{m}_{32}^{(3)}
\end{array}\right)^{T}=\left(\begin{array}{lll}
0 & 1 & 0
\end{array}\right)^{T}
$$

and hence

$$
m^{(2)}=\left(\begin{array}{ll}
\widetilde{m}_{11}^{(3)} & \widetilde{m}_{13}^{(3)} \\
\widetilde{m}_{31}^{(3)} & \widetilde{m}_{33}^{(3)}
\end{array}\right)
$$

solves the RHP

$$
\left\{\begin{array}{l}
m_{+}^{(2)}=m_{-}^{(2)}\left(\begin{array}{cc}
1-s_{2} & s_{2}\left(\varphi z^{n}\right)^{-1} \\
-s_{2} \varphi z^{n} & 1+s_{2}
\end{array}\right), \quad z \in \Sigma, \\
m^{(2)} \rightarrow I \quad \text { as } z \rightarrow \infty,
\end{array}\right.
$$

which is again the (equivalent) RHP for PIII.

The analogy with solitons is particularly clear if we consider $v^{(3)}$ in the edge scaling limit,

$$
n_{j}=2 \tau+t_{j} \tau^{1 / 3}, \quad j=1,2 \quad ; \quad t_{1}<t_{2},
$$

as $\tau \rightarrow \infty$. Then

$$
v^{(3)}\left(-1+\frac{2 i u}{\tau^{1 / 3}}\right) \mapsto \widehat{v}^{(3)}(u)=\left(\begin{array}{ccc}
1-s_{2} & s_{2} e^{2 i \theta_{1}} & s_{2} e^{2 i \theta_{2}} \\
-s_{1} e^{-2 i \theta_{1}} & 1+s_{1} & s_{1} e^{-2 i\left(\theta_{1}-\theta_{2}\right)} \\
-\left(s_{2}-s_{1}\right) e^{-2 i \theta_{2}} & \left(s_{2}-s_{1}\right) e^{2 i\left(\theta_{1}-\theta_{2}\right)} & 1+s_{2}-s_{1}
\end{array}\right)
$$

on the real line, where

$$
\theta_{j}=\frac{4}{3} u^{3}+t_{j} u, \quad j=1,2 .
$$

In addition to varying $s_{1}, s_{2}$, we can now vary $t_{1}, t_{2}$. In particular, we can follow the trajectory of the solution of the RHP as $t_{2}$ moves from $t_{1}$ to $\infty$. As $t_{2} \rightarrow t_{1}$, the solution becomes Painlevé II (PII) and as $t_{2} \rightarrow \infty$, it gives to another solution of PII, but now with a phase shift (see [2]). It is this behavior of P-tons, in particular, that is reminiscent of soliton interactions.

\section{Colored permutations}

First, the definition:

Let $\pi$ be an $m$-colored permutation (see, e.g., [32]), and assume the colors are indexed by $0,1, \ldots m-$

1. Let $S$ be a subsequence of length $l$ of $\pi$ which is a union of monochromatic increasing subsequences; 
let $k_{i}$ be the number of these sequences having color $i$, and set $k=\sum_{i} k_{i}$. Note that the monochromatic increasing subsequences may be empty, but the color of empty subsequences still matters. We assign to $S$ the following score:

$$
m l+\left(\begin{array}{c}
k+1 \\
2
\end{array}\right)+\sum_{0 \leq i \leq m-1}\left(i k_{i}-m\left(\begin{array}{c}
k_{i}+1 \\
2
\end{array}\right)\right)
$$

Now, let $l_{k}(\pi)$ be the maximum score over all unions of $k$ monochromatic increasing subsequences (note $\left.l_{0}(\pi)=0\right)$. We then define

$$
\lambda_{k}(\pi):=l_{k}(\pi)-l_{k-1}(\pi)
$$

Lemma 7.1. Let $\lambda_{k}^{(i)}(\pi)$ be the partition associated to just the $i$-colored subsequence of $\pi$. Then $\lambda_{k}(\pi)-$ $k$ is simply the $k$ th largest of the numbers $m\left(\lambda_{j}^{(i)}(\pi)-j\right)+i$. Moreover, if $\pi$ has length $n$, then $\lambda_{k}(\pi)$ is a partition of $m n$.

Proof. Fix a composition $k_{i}$, and consider the largest score associated to that composition. Clearly, we can maximize the score for each color independently; we thus obtain:

$$
m l+\left(\begin{array}{c}
k+1 \\
2
\end{array}\right)+\sum_{0 \leq i \leq m-1}\left(i k_{i}-m\left(\begin{array}{c}
k_{i}+1 \\
2
\end{array}\right)\right)=\left(\begin{array}{c}
k+1 \\
2
\end{array}\right)+\sum_{0 \leq i \leq m-1} \sum_{1 \leq j \leq k_{i}}\left(m\left(\lambda_{j}^{(i)}(\pi)-j\right)+i\right) .
$$

Now, for a fixed value of $k$, this is clearly maximized when the values $m\left(\lambda_{j}^{(i)}(\pi)-j\right)+i$ occurring in the sum are chosen to be as large as possible. Plugging the resulting value of $l_{k}(\pi)$ into the formula for $\lambda_{k}(\pi)$, we obtain the first claim.

Note that the numbers $m\left(\lambda_{j}^{(i)}(\pi)-j\right)+i$ are all different (the congruence class modulo $m$ depends on the color, and the numbers are distinct within a given color). Furthermore, we readily verify that for each congruence class, the number of negative numbers not occurring in the set is equal to the number of nonnegative numbers occurring in the set. We thus conclude that $\lambda_{k}(\pi)$ is indeed a partition. It remains to verify that $\sum_{k} \lambda_{k}(\pi)=m n$; in other words, $l_{k}(\pi)=m n$ for $k$ sufficiently large. Choose $k$ such that $\pi$ is a union of $k$ increasing subsequences, and consider $l_{m k}(\pi)$. We readily verify that the term

$$
\sum_{0 \leq i \leq m-1}\left(i k_{i}-m\left(\begin{array}{c}
k_{i}+1 \\
2
\end{array}\right)\right)
$$

is maximized when all $k_{i}$ are equal to $k$, and thus the optimal score differs from $m n$ by

$$
\left(\begin{array}{c}
m k+1 \\
2
\end{array}\right)+\sum_{0 \leq i \leq m-1}\left(i k-m\left(\begin{array}{c}
k+1 \\
2
\end{array}\right)\right)=0
$$

Remark 7.2. An alternate approach is to define $\lambda_{k}(\pi)$ via the Schensted correspondence for rim-hook permutations given in [34], at which point the lemma follows immediately. The fact that the rim-hook correspondence splits into $m$ ordinary correspondences gives the increasing subsequence interpretation above. 
Now, suppose we choose $n$ randomly according to a Poisson law of mean $m t^{2}$, and then choose an $m$-colored permutation of length $n$ at random. Equivalently, take $m$ independent Poisson processes in the unit square (one for each color), and convert the resulting point set to a colored permutation. We thus see that the resulting random partitions $\lambda_{j}^{(i)}(\pi)$ are independent, and are all distributed according to the law for ordinary permutations. In particular, we obtain the following correlation kernel:

$$
\mathrm{S}^{(m)}(a, b)=\sum_{k \geq 1}\left(\varphi^{-1}\right)_{(a+k) / m} \varphi_{(b+k) / m}
$$

where

$$
\varphi(z)=e^{t\left(z-z^{-1}\right)}
$$

(Recall from Corollary 2.10 that $\varphi_{a}$ and $\left(\varphi^{-1}\right)_{a}$ are 0 for $a$ non-integral.)

Now by using Corollary 2.10 and Theorem 3.1 for the convergence of moments for the ordinary permutations, we obtain the convergence of moments for $\lambda_{k}$ 's in the colored permutation setting. More precisely, as in (3.1), there is a limiting distribution $F^{\text {color }(m)}$ such that

$$
\lim _{N \rightarrow \infty} \mathbb{E}_{N}^{\text {color }(m)}\left(\prod_{j=1}^{k}\left(\frac{\lambda_{j}-2 \sqrt{m N}}{m^{2 / 3}(m N)^{1 / 6}}\right)^{a_{j}}\right)=\mathbb{E}^{\text {color }(m)}\left(x_{1}^{a_{1}} \cdots x_{k}^{a_{k}}\right)
$$

where $\mathbb{E}_{N}^{c o l o r(m)}$ denotes the expectation with respect to the natural counting measure on the colored permutations (see [32]), and $\mathbb{E}^{\text {color }(m)}$ is the expectation with respect to $F^{\operatorname{color}(m)}$. The function $F^{\text {color }(m)}\left(x_{1}, \cdots, x_{k}\right)$ has the following meaning in terms of GUE. Take $m$ random GUE matrices of size $N$ at random, then superimpose their eigenvalues. We denote the largest of those superimposed numbers by $z_{1}(N)$, the second largest by $z_{2}(N)$, and so on. Then $F^{\operatorname{color}(m)}\left(x_{1}, \cdots, x_{k}\right)$ is the limiting distribution of $z_{1}, \cdots, z_{k}$ as $N \rightarrow \infty$, after appropriate centering and scaling.

A number of other statistical systems which are currently of interest can also be analyzed by the methods of this paper. In particular, we have in mind the random word problem [35, 24, 22, 23, certain 2-dimensional growth models [25], and also the so-called "digital boiling model" 20].

For example, in the growth model considered by Johansson in [25], let $\sigma=\cup_{j=1}^{k} \sigma_{j}$ be a union of $k$ disjoint increasing paths $\sigma_{j}$ in the model. Let $L^{(k)}(\sigma)$ be the sum of the lengths of the paths $\sigma_{j}$, and let $L^{(k)}=\max _{\sigma} L^{(k)}(\sigma)$. We define $\lambda_{k}=L^{(k)}-L^{(k-1)}$. The joint probability distribution for $\lambda_{1}, \cdots, \lambda_{k}$ can be obtained [25] by various differentiations of $\operatorname{det}\left(1+\sum_{j=1}^{k} s_{j} \chi_{\left[n_{j} . n_{j-1}\right)} \mathrm{S}\right)$ with respect to $s_{1}, \cdots, s_{k}$ as in (1.12) with $\varphi$ now given by $\varphi(z)=(1+\sqrt{q} z)^{M}\left(1+\sqrt{q} z^{-1}\right)^{-N}$. But now by Theorem 2.12, $\operatorname{det}\left(1+\sum_{j=1}^{k} s_{j} \chi_{\left[n_{j} . n_{j-1}\right)} \mathrm{S}\right)$ can be expressed in terms of the determinant of an integrable operator as in (2.54). This opens up the possibility for the asymptotic analysis of the convergence of moments for the joint distribution. However, the associated RHP has a new feature, namely the weight function is non-real, which has not yet been addressed in general (however, see [27]). There are similar formulae for random words and digital boiling.

\section{References}


[1] M. Abramowitz and I. Stegun. Handbook of Mathematical Functions. Dover Publications, New York, 1965.

[2] J. Baik, P. Deift, and A. Its. in preparation.

[3] J. Baik, P. Deift, and K. Johansson. On the distribution of the length of the longest increasing subsequence of random permutations. J. Amer. Math. Soc., 12(4):1119-1178, 1999.

[4] J. Baik, P. Deift, and K. Johansson. On the distribution of the length of the second row of a Young disgram under Plancherel measure. math.CO/9901118; http://xxx.lanl.gov/abs/, to appear in Geom. Funct. Anal.

[5] E. Basor and H. Widom. On a Toeplitz determinant identity of Borodin and Okounkov. math.FA/9809088; http://xxx.lanl.gov/abs/.

[6] R. Beals and R. Coifman. Scattering and inverse scattering for first order systems. Comm. Pure Appl. Math., 37:39-90, 1984.

[7] A. Borodin and A. Okounkov. A Fredholm determinant formula for Toeplitz determinants. math.CA/9907165; http://xxx.lanl.gov/abs/.

[8] A. Borodin, A. Okounkov, and G. Olshanski. On asymptotics of Plancherel measures for symmetric groups. J. Amer. Math. Soc., 13(3):481-515, 2000.

[9] A. Böttcher. On the determinant formulas by borodin, okounkov, baik, deift, and rains. math.FA/0101008; http://xxx.lanl.gov/abs/.

[10] A. Böttcher. One more proof of the borodin-okounkov formula for toeplitz determinants. math.FA/0012200; http://xxx.lanl.gov/abs/.

[11] K. Clancey and I. Gohberg. Factorization of Matrix Functions and Singular Integral Operators. Birkhäuser, 1981.

[12] P. Deift. Integrable operators. Amer. Math. Soc. Transl. Ser. 2, 189:69-84, 1999.

[13] P. Deift, T. Kriecherbauer, K. McLaughlin, S. Venakides, and X. Zhou. Strong asymptotics of orthogonal polynomials with respect to exponential weights. Comm. Pure Appl. Math., 52(12):1491$1552,1999$.

[14] P. Deift, T. Kriecherbauer, K. McLaughlin, S. Venakides, and X. Zhou. Uniform asymptotics for polynomials orthogonal with respect to varying exponential weights and applications to universality questions in random matrix theory. Comm. Pure Appl. Math., 52(11):1335-1425, 1999.

[15] P. Deift, S. Venakides, and X. Zhou. New results in small dispersion KdV by an extension of the steepest descent method for Riemann-Hilbert problems. Internat. Math. Res. Notices, 6:285-299, 1997. 
[16] P. Deift and X. Zhou. A steepest descent method for oscillatory Riemman-Hilbert problems. Asymptotics for the MKdV equation. Ann. of Math., 137:295-368, 1993.

[17] P. Deift and X. Zhou. Asymptotics for the Painlevé II equation. Comm. Pure Appl. Math., 48:277-337, 1995.

[18] A. Fokas, U. Mugan, and X. Zhou. On the solvability of Painlevé I, III and V. Inverse Problems, 8:757-785, 1992.

[19] I. Gessel. Symmetric functions and P-recursiveness. J. Combin. Theory Ser. A, 53:257-285, 1990.

[20] J. Gravner, C. Tracy, and H. Widom. Limit theorems for height fluctuations in a class of discrete space and time growth models. math.PR/0005133; http://xxx.lanl.gov/abs/.

[21] A. Its, A. Izergin, V. Korepin, and N. Slavnov. Differential equations for quantum correlation functions. Internat. J. Modern Phys. B, 4(5):1003-1037, 1990.

[22] A. Its, C. Tracy, and H. Widom. Random words, Toeplitz determinants and integrable systems. I. math.CO/9909169; http://xxx.lanl.gov/abs/.

[23] A. Its, C. Tracy, and H. Widom. Random words, Toeplitz determinants and integrable systems. II. nlin.SI/9909169; http://xxx.lanl.gov/abs/.

[24] K. Johansson. Discrete orthogonal polynomial ensembles and the Plancherel measure. math.CO/9906120; http://xxx.lanl.gov/abs/.

[25] K. Johansson. Shape fluctuations and random matrices. math.CO/9903134 ; http://xxx.lanl.gov/abs/.

[26] K. Johansson. The longest increasing subsequence in a random permutation and a unitary random matrix model. Math. Res. Lett., 5(1-2):63-82, 1998.

[27] S. Kamvissis, K. McLaughlin, and P. Miller. Semiclassical soliton ensembles for the focusing nonlinear Schrödinger equation. preprint, 2000.

[28] A. Okounkov. Infinite wedge and random partitions. math.RT/9907127; http://xxx.lanl.gov/abs/.

[29] A. Okounkov. Random matrices and random permutations. math.CO/9903176 ; http://xxx.lanl.gov/abs/.

[30] E. Rains. A mean identity for longest increasing subsequence problems. math.CO/0004082 ; http://xxx.lanl.gov/abs/.

[31] E. M. Rains. Correlation functions for symmetrized increasing subsequences. math.CO/0006097 ; http://xxx.lanl.gov/abs/. 
[32] E. M. Rains. Increasing subsequences and the classical groups. Electron. J. Combin., 5(1):R12, 1998.

[33] B. Simon. Trace ideals and their applications, volume 35 of London Mathematical Society lecture note series. Cambridge, New York, NY, 1979.

[34] D. W. Stanton and D. E. White. A Schensted algorithm for rim hook tableaux. J. Combin. Theory Ser. A, 40:211-247, 1985.

[35] C. Tracy and H. Widom. On the distribution of the lengths of the longest monotone subsequences in random words. math.CO/9904042; http://xxx.lanl.gov/abs/.

[36] C. Tracy and H. Widom. Level-spacing distributions and the Airy kernel. Comm. Math. Phys., 159:151-174, 1994.

[37] E. Whittaker and G. Watson. A Course of Modern Analysis. Cambridge University Press, Cambridge, 4th edition, 1927. 